\title{
Betti numbers of finite volume orbifolds
}

\author{
IDDO SAMET
}

\begin{abstract}
We prove that the Betti numbers of a negatively curved orbifold are linearly bounded by its volume, generalizing a theorem of Gromov that establishes this bound for manifolds. An immediate corollary is that Betti numbers of a lattice in a rank-one Lie group are linearly bounded by its co-volume.
\end{abstract}

$53 \mathrm{C} 20$

\section{Introduction}

Let $X$ be a Hadamard manifold, ie, a connected, simply connected, complete Riemannian manifold of non-positive curvature normalized such that $-1 \leq K \leq 0$. Let $\Gamma$ be a discrete subgroup of $\operatorname{Isom}(X)$. If $\Gamma$ is torsion-free then $X / \Gamma$ is a Riemannian manifold. If $\Gamma$ has torsion elements, then $X / \Gamma$ has a structure of an orbifold. In both cases, the Riemannian structure defines the volume of $X / \Gamma$.

An important special case is that of symmetric spaces $X=K \backslash G$, where $G$ is a connected semisimple Lie group without center and with no compact factors, and $K$ is a maximal compact subgroup. Then $X$ is non-positively curved, and $G$ is the connected component of $\operatorname{Isom}(X)$. In this case, $X / \Gamma$ has finite volume iff $\Gamma$ is a lattice in $G$. The curvature of $X$ is non-positive if $\operatorname{rank}(G) \geq 2$, and is negative if $\operatorname{rank}(G)=1$.

In many cases, the volume of negatively curved manifold controls the complexity of its topology. A manifestation of this phenomenon is the celebrated theorem of Gromov $[3 ; 10]$ stating that if $X$ has sectional curvature $-1 \leq K<0$ then

$$
\sum_{i=0}^{n} b_{i}(X / \Gamma) \leq C_{n} \cdot \operatorname{vol}(X / \Gamma),
$$

where $b_{i}$ are Betti numbers w.r.t. coefficients in any field, and $C_{n}$ is a constant depending only on $n=\operatorname{dim}(X)$. This inequality also holds when $-1 \leq K \leq 0$ if $X$ is analytic and has no Euclidean factors.

It should be noted, that since $X$ is a $K(\Gamma, 1)$, the $b_{i}$ are the Betti number of the homology of the group $\Gamma$. 
In certain cases, volume imposes much stronger restriction on topology. Namely, it was proved by Gelander [8] that if $X$ is a symmetric space of non-compact type and $\Gamma$ is a non-uniform torsion-free arithmetic lattice then $X / \Gamma$ is homotopy-equivalent to a simplicial complex whose complexity is restricted: the number of vertices in this complex is bounded by a constant (depending on $X$ ) times the volume of $X / \Gamma$, and the valence of each vertex is bounded by a constant depending only on $X$. This equivalence immediately implies Gromov's Theorem. By Margulis's Arithmeticity Theorem, if $\operatorname{rank}(X) \geq 2$ then every lattice $\Gamma$ is arithmetic, and thus Gelander's theorem holds for all such spaces.

Gelander has also proved that if $\Gamma$ torsion-free, then $\Gamma$ has a presentation for which the number of generators and the total length of the relations is bounded linearly by the volume of $X / \Gamma$ (with constants depending on $X$ ). Recently, Gelander [9] extended this result and proved that even for lattices with torsion, the minimal number of generators of $\Gamma$ is bounded from above by a constant (depending on $G$ ) times the volume of $X / \Gamma$. In particular, this provides a linear bound to the first Betti number of $\Gamma$.

The main theorem proved in this paper is a generalization of Gromov's theorem to the case where $\Gamma$ has torsion, in other words, to orbifolds of the form $X / \Gamma$. We prove:

Theorem 1.1 Let $X$ be a Hadamard manifold of dimension $n$ with sectional curvature $-1 \leq K<0$. Then for every discrete group $\Gamma<\operatorname{Isom}(X)$,

$$
\sum_{i} \operatorname{rk} H_{i}(X / \Gamma, A) \leq C_{n} \cdot \operatorname{vol}(X / \Gamma),
$$

where the coefficient ring $A$ is an integral domain of characteristic 0 , and $C_{n}$ is a constant depending only on $n$.

There are several (co)-homology theories for orbifolds that aim at capturing the homology of the space, as well as that of the group $\Gamma$. Our technique is mainly geometric; we therefore treat $X / \Gamma$ as a topological quotient space, and the homology is that of this space. Furthermore, the action of finite subgroups of $\Gamma$ introduces torsion to homology groups, which may depend on the structure of these finite groups. Since we are not able to bound the size of finite groups by the orbifold volume, our result is restricted to homology with coefficients in an integral domain with characteristic 0 .

The case $A=\mathbb{Q}$ is of particular importance. The homology of the stabilizers in $G$ of points of $X$ is trivial, because stabilizers are finite groups. It follows that $H_{i}(X / \Gamma, \mathbb{Q})=H_{i}(\Gamma, \mathbb{Q})$ (see eg [6, page 174]). Hence, we can restate (2) as a bound 
on Betti numbers (with rational coefficients) of the group $\Gamma$ :

$$
\sum_{i} b_{i}(\Gamma, \mathbb{Q}) \leq C_{n} \cdot \operatorname{vol}(X / \Gamma) .
$$

Theorem 1.1 and Gromov's theorem can be rephrased as an asymptotic statement: if $\Gamma_{n}$ is a sequence of groups of isometries acting discretely on $X$ (with the appropriate assumptions) then the growth of $b_{i}\left(\Gamma_{n}\right)$ is at most linear in $\operatorname{vol}\left(X / \Gamma_{n}\right)$.

A theorem of Lück [12] describes the asymptotic behavior of a nested sequence $\left(\Gamma_{n}\right)_{n \geq 1}$ of normal finite index subgroups of $\Gamma$, assuming that $X / \Gamma$ is compact and $\bigcap \Gamma_{n}=1$. In this case,

$$
\lim _{n \rightarrow \infty} \frac{b_{i}\left(X / \Gamma_{n}\right)}{\operatorname{vol}\left(X / \Gamma_{n}\right)}=\beta_{i}^{(2)}(X ; \Gamma)
$$

where the right-hand side is the $L^{2}$-Betti number. Hence, the growth is linear exactly when $\beta_{i}^{(2)}(X ; \Gamma)$ is non-zero. It was proved by Olbrich [15] that if $X$ is a symmetric space, then $\beta_{i}^{(2)}$ is zero unless, perhaps, $i=\operatorname{dim}(X) / 2$.

In a recent joint work with Abert, Bergeron, Biringer, Gelander, Nikolov and Raimbault [2; 1] we prove (3) holds for any sequence of uniform irreducible lattices $\left(\Gamma_{n}\right)_{n \geq 1}$ (possibly with torsion) in a semisimple Lie group $G$, assuming that $\operatorname{vol}\left(X / \Gamma_{n}\right) \rightarrow \infty$ and that $G$ has property (T) and rank $\geq 2$. However, one cannot expect (3) to hold for rank-one locally symmetric spaces (whether manifolds or orbifolds). For instance, if $\Gamma$ is a uniform lattice in $\operatorname{Isom}\left(\mathbb{H}^{n}\right)$ that surjects on the free group of rank 2 , then finite index subgroups of $\Gamma$ corresponding to subgroups of $\mathbb{Z} * \mathbb{Z}$ have first Betti numbers that grow linearly with volume, whereas $\beta_{1}^{(2)}=0$.

\section{Characteristic $p$}

It is natural to whether Theorem 1.1 holds for coefficients with positive characteristic, and particularly for coefficients in the field $\mathbb{F}_{p}$. The author does not know of any counterexample in this case. In fact, there is but a single place in the proof that relies on the characteristic assumption, namely Proposition 4.11. Proving this proposition for coefficients in $\mathbb{F}_{p}$ boils down to the problem of uniformly bounding the $\mathbb{F}_{p}$-Betti numbers of a spherical orbifold, as posed in the following question:

Does there exist a constant $D$ depending on $k$ (and possibly on $p$ ?) such that for every finite group $G$ acting linearly on a sphere $S^{k}$, and for every $i, b_{i}\left(S^{k} / G, \mathbb{F}_{p}\right) \leq D$ ? 
More precisely, an affirmative answer to the question for spheres of dimensions $\leq$ $n-1$ would imply that Theorem 1.1 holds with coefficients in $\mathbb{F}_{p}$ for manifolds of dimension $n$. In particular, we can obtain the following partial results:

(1) The theorem holds with $\mathbb{F}_{p}$ coefficients for manifolds of dimensions 2 or 3 (with a constant $C_{n}$ independent of $p$ ). This is because - up to homeomorphismthere are only finitely many possible quotients of 1 -spheres and 2 -spheres by finite groups. ${ }^{1}$

(2) If $p$ does not divide the order of any finite subgroup of $\Gamma$, then the inequality (2) holds for $A=\mathbb{F}_{p}$ (with the constant $C_{n}$ of the characteristic 0 case). This follows from the fact that $b_{i}\left(S^{k} / G, \mathbb{F}_{p}\right) \leq 1$ whenever $p$ is prime to the order of $G$ (see remark at the end of Section 4 ).

\section{Acknowledgements}

This research was done as part of my Ph.D. studies at the Hebrew University, under the supervision of Prof. Tsachik Gelander. It is my pleasure to thank Tsachik for his support, encouragement and advice. I also thank the referee for carefully reading the manuscript, and for his invaluable suggestions for improving the exposition. The research was supported by the European Research Council (ERC) grant of Prof. Gelander / agreement 203418.

\section{Preliminaries}

\subsection{Notation}

Let $X$ be an $n$-dimensional Hadamard manifold, ie, a connected, simply connected complete Riemannian manifold of non-positive sectional curvature. We assume that the curvature is bounded and that the metric is normalized such that $-1 \leq K \leq 0$. If $K<0$ we say the manifold is negatively curved. Most of our results apply to this case.

2.1.1 We introduce notation and review some facts about isometries of Hadamard spaces. A standard reference for this is [5, Chapter II.6] or [3, Section 6].

Let $\gamma$ be an isometry of $X$. The displacement function $d_{\gamma}: X \rightarrow \mathbb{R}^{\geq 0}, d_{\gamma}(x)=$ $d(\gamma x, x)$, is a convex function in the sense that for every geodesic $c: \mathbb{R} \rightarrow X, d_{\gamma} \circ c$ is a convex function. The set of minimal displacement is defined:

$$
\operatorname{Min}(\gamma)=\left\{x \in X: d_{\gamma}(x)=\inf d_{\gamma}\right\}
$$

${ }^{1}$ The underlying space of a 1-dimensional spherical orbifold is 1-sphere or an interval, and the underlying space of a 2 -dimensional spherical orbifold is a $2-$ sphere, a disc, or a projective plane. 
It is a closed and convex subset of $X$.

An isometry of $X$ is elliptic if it fixes a point in $X$, hyperbolic if it does not fix a point in $X$ but $d_{\gamma}$ attains minimum in $X$, and parabolic otherwise. The first two types are called semisimple. An isometry is elliptic (resp. hyperbolic, parabolic) iff any a positive power of it is elliptic (resp. hyperbolic, parabolic).

If $\gamma$ is a non-trivial elliptic isometry then $\operatorname{Min}(\gamma)$ is a complete totally geodesic submanifold of lower dimension in $X$. If $\gamma$ is hyperbolic and $K<0$ then $\operatorname{Min}(\gamma)$ is a geodesic - the axis of $\gamma$-on which $\gamma$ acts by translations. If $\gamma$ is a parabolic isometry then it fixes a point in the boundary of points at infinity. Explicitly, there is a geodesic ray $c: \mathbb{R}^{+} \rightarrow X$ such that $c$ and $\gamma c$ are asymptotic, ie, $d(c(t), \gamma c(t))$ is uniformly bounded. If $K<0$ then $\gamma$ fixes a unique point at infinity.

Let $C$ be a closed convex subset of $X$, and let $x \in X$. Since $X$ has non-positive curvature, there is a unique point $\pi_{C}(x) \in C$ that is closest to $x$ in $C$. This is called the projection to $C$. The projection is distance decreasing, ie, $d\left(\pi_{C}(x), \pi_{C}(y)\right) \leq$ $d(x, y)$. Now suppose $C$ is $\gamma$-invariant. Then $\gamma \pi_{C}(x)=\pi_{C}(\gamma x)$. It follows that $d_{\gamma}\left(\pi_{C}(x)\right) \leq d_{\gamma}(x)$. In particular, $\operatorname{Min}(\gamma) \cap C$ is non-empty.

If $\gamma_{0}, \gamma_{1}$ are commuting semisimple isometries, then $\gamma_{i}$ keeps $\operatorname{Min}\left(\gamma_{1-i}\right)$ invariant $(i=0,1)$. It follows that $\operatorname{Min}\left(\gamma_{0}\right) \cap \operatorname{Min}\left(\gamma_{1}\right)$ is non-empty, and kept invariant by both isometries. More generally, if $A$ is a set of commuting semisimple isometries then $\bigcap_{\gamma \in A} \operatorname{Min}(\gamma)$ is non-empty and $A$-invariant. In particular, if $K<0$ then two commuting isometries have the same axis.

2.1.2 Let $\Gamma$ be a group of isometries of $X$ acting properly discontinuously, that is, a subgroup of Isom $(X)$ that is discrete with respect to the compact-open topology. If $\Gamma$ is torsion-free, then $X / \Gamma$ has a structure of Riemannian manifold. More generally, $\Gamma$ may have finite point-stabilizers, and $X / \Gamma$ is endowed a structure of a Riemannian orbifold. An orbifold has an atlas of maps locally identifying it with a quotient of an open set in $X$ by a finite group of isometries. Orbifolds were originally introduced and studied by Satake [17] who named them V-manifolds.

2.1.3 We have the canonical projection $\pi: X \rightarrow X / \Gamma$. Let inj-rad $(x), x \in X / \Gamma$, be the local injectivity radius at $x$. Let us denote $d_{\Gamma}(x)=\inf _{\gamma \in \Gamma \backslash\{1\}} d_{\gamma}(x)$. We recall that $\operatorname{inj}-\operatorname{rad}(x)=\frac{1}{2} d_{\Gamma}(\tilde{x})$, with $\pi(\tilde{x})=x$. The $\varepsilon$-thick part of $X / \Gamma$ is defined:

$$
(X / \Gamma)_{\geq \varepsilon}=\left\{x \in X / \Gamma: \operatorname{inj}-\operatorname{rad}(x) \geq \frac{\varepsilon}{2}\right\}
$$

It is the image of the set $\left\{x \in X: d_{\Gamma}(x) \geq \varepsilon\right\}$ under the canonical projection.

We remark that if $X$ is a point, its injectivity radius is infinity. 
2.1.4 We will make all volume calculations using discrete estimates.

Say a set $\mathcal{N} \subset X$ is $\delta$-discrete if $d(x, y) \geq \delta$ for $x \neq y \in \mathcal{N}$. For $\varepsilon>0$ we define $\varepsilon$-ess-vol $(X / \Gamma)$ as the supremal cardinality of a $2 \varepsilon$-discrete set of points $\mathcal{N}$ in the $2 \varepsilon$-thick part of $X / \Gamma$. This is the supremum of the number of disjoint injected balls of radius $\varepsilon$. It is reasonable to set $\varepsilon$-ess-vol(point) $=1$; this is consistent with definitions for positive dimension. This definition is introduced in [3, Section 10.4].

Clearly, $\varepsilon$-ess- $\operatorname{vol}(X / \Gamma)$ is non-decreasing as $\varepsilon$ decreases. In the case $\operatorname{dim} X>0$, it is easy to verify the useful inequality

$$
k \cdot \varepsilon \text {-ess-vol }(X / \Gamma) \leq \frac{\varepsilon}{k} \text {-ess-vol }(X / \Gamma) .
$$

Since curvature is non-positive, a ball of radius $\varepsilon$ in $X$ has volume $\geq V(\varepsilon, n)$ - the volume of a ball of radius $\varepsilon$ in Euclidean $n$-space. Thus we have:

$$
\varepsilon \text {-ess-vol }(X / \Gamma) \leq V(\varepsilon, n)^{-1} \cdot \operatorname{vol}(X / \Gamma)
$$

We will utilize these two inequalities freely without further reference.

2.1.5 For a point $x \in X$, let $\Gamma_{x}$ be the stabilizer of $x$ in $\Gamma$. A subgroup of $\Gamma$ is finite if and only if it is contained in $\Gamma_{x}$, for some $x \in X$.

For a submanifold $Y \subseteq X$, we denote:

$$
\Gamma_{Y}=\{\gamma \in \Gamma: \gamma Y=Y\} \quad \text { and } \quad \Gamma_{Y}^{1}=\left\{\gamma \in \Gamma_{Y}:\left.\gamma\right|_{Y}=1\right\}
$$

The latter is a finite group.

If $\Gamma_{Y}^{1}$ is not trivial, then $\Gamma_{Y}$ cannot be identified as a subgroup of $\operatorname{Isom}(Y)$. However, the action of $\Gamma_{Y}$ on $Y$ factors through $\Gamma_{Y} / \Gamma_{Y}^{1}$. In this case, the terms $Y / \Gamma_{Y}$ and $d_{\Gamma_{Y}}$, and consequentially inj-rad and $\varepsilon$-ess-vol $\left(Y / \Gamma_{Y}\right)$, will refer to-by abuse of notation-the action $\Gamma_{Y} / \Gamma_{Y}^{1}$ on $Y$.

\subsection{Margulis Lemma}

We denote $\Delta_{\varepsilon}(x)=\left\{\gamma \in \Gamma: d_{\gamma}(x)<\varepsilon\right\}$ and $\Gamma_{\varepsilon}(x)=\left\langle\Delta_{\varepsilon}(x)\right\rangle$. The following statement about groups generated by "small motions" is an amalgamation of the Margulis Lemma [3] and the Jordan Theorem [11].

Theorem 2.1 Let $X$ be a Hadamard manifold of dimension $n$ with $-1 \leq K \leq 0$. There are constants depending only on $n, \varepsilon_{n}>0$ and $m_{n} \in \mathbb{N}$ such that for every discrete subgroup $\Gamma<\operatorname{Isom}(X)$ and every $x \in X, \Gamma_{\varepsilon_{n}}(x)$ contains a normal nilpotent subgroup $N$ of index $\leq m_{n}$. If $\Gamma_{\varepsilon_{n}}(x)$ is finite, $N$ is in fact abelian. 
We henceforth refer to the nilpotent subgroup stipulated by this theorem as "the normal nilpotent subgroup of $\Gamma_{\varepsilon}(x)$ ".

Remark The proof of the Margulis Lemma (cf [3, Section 8.3]) is based on the fact that there are generators of $N$ in a neighborhood of identity in which commutators are contracting with respect to some norm. It follows from this that for every $\delta>0$ there is $K=K(\delta, n)$ such that if $\gamma$ is a $k$-fold commutator of these generators with $k \geq K$ then $d_{\gamma}(x)<\delta$.

The following lemma is proved in [3, Lemma 7.4].

Lemma 2.2 Let $N$ be a nilpotent group of isometries acting on a closed convex subset of $X$. Let $N_{s}$ be the set of semisimple isometries in $N$. Then $N_{s}$ is a normal subgroup of $N$, and $\bigcap_{\gamma \in N} \operatorname{Min}(\gamma)$ is a non-empty $N$-invariant set.

Lemma 2.3 Let $0<\varepsilon<\varepsilon_{n}$, and let $N$ be the normal nilpotent subgroup of index $\leq m_{n}$ in $\Gamma_{\varepsilon}(x)$. Then $N$ is generated by elements in $\Delta_{2 m_{n} \varepsilon}(x)$. If $\Gamma_{\varepsilon}(x)$ is infinite then there is an element of infinite order in $N \cap \Delta_{2 m_{n} \varepsilon}(x)$. Furthermore, if $\Gamma_{\varepsilon}(x)$ contains parabolic elements then this element can be taken to be parabolic.

Proof Recall that if $G$ is a group generated by a set of elements $S$, and $H \leq G$ is a subgroup of index $r$, then $H$ is generated by words of length $<2 r$ in the generators $S$. Indeed, if $T=\left\{t_{1}, \ldots, t_{r}\right\}$ is a transversal (ie, a set of representatives of right cosets) of $H$ in $G$, then $H$ is generated by $T S T^{-1} \cap H$. Moreover, if $T$ is a Schreier transversal (w.r.t. the generating set $S$ ) then its elements have length at most $r-1$ (for the last two statements, see eg [13, Section 2.3]). Hence, $H$ is generated by words of length $2(r-1)+1<2 r$.

Let $N$ be the normal nilpotent subgroup of $\Gamma_{\varepsilon}(x)$. Since $[G: N] \leq m_{n}, N$ is generated by words of length $<2 m_{n}$ with respect to the generators of $\Gamma_{\varepsilon}(x)$. Hence it is generated by elements in $\Delta_{2 m_{n} \varepsilon}(x)$.

Let $N_{s}$ be the set of semisimple elements in $N$. By Lemma 2.2, $N_{s}$ is a subgroup, and $K=\bigcap_{\gamma \in N_{s}} \operatorname{Min}(\gamma)$ is non-empty convex and $N$-invariant. Notice that every elliptic element in $N$ fixes $K$. Since $N$ is infinite, it cannot fix $K$ pointwise, and thus cannot be generated by elliptic elements. Thus, at least one element in $N \cap \Delta_{2 m_{n} \varepsilon}(x)$ has infinite order. If $\Gamma_{\varepsilon}(x)$ contains a parabolic element, then so does $N$. In this case, at least one element in $N \cap \Delta_{2 m_{n} \varepsilon}(x)$ is parabolic, otherwise $N=N_{s}$. 


\subsection{Elliptic isometries}

For an elliptic isometry $\gamma$ we write $\operatorname{Fix}(\gamma)$ for $\operatorname{Min}(\gamma)$. Let $\Gamma$ be a group of isometries acting discretely on $X$. We say $Y$ is a singular submanifold if it equals $\bigcap_{\gamma \in A} \operatorname{Fix}(\gamma)$ for a set $A \subset \Gamma$ of elliptic elements. We do not exclude the trivial case $Y=X$.

Let $Y, Y^{\prime}$ be two totally geodesic submanifolds in $X$. If there exists $\gamma \in \Gamma$ such that $Y=\gamma Y^{\prime}$ we say that $Y, Y^{\prime}$ are conjugate. Clearly, if $Y$ is conjugate to a singular submanifold, then $Y$ itself is a singular submanifold.

Definition 2.4 An isometry $g \in G=\mathrm{SO}(n)$ of $\mathbb{R}^{n}$ is $s$-stable $(s \in \mathbb{N})$ if $C_{G}\left(g^{i}\right)=$ $C_{G}(g)$ (centralizer in $G$ ) and $\operatorname{Fix}(g)=\operatorname{Fix}\left(g^{i}\right)$ for $i=1, \ldots, s$. An elliptic isometry $\gamma \in \operatorname{Isom}(X)$ is $s$-stable if the isometry induced on the tangent bundle of a fixed point is $s$-stable.

A singular submanifold $Y$ is $s$-stable (w.r.t. to $\Gamma$ ) if there exists a subset $A \subset \Gamma$, such that every $\gamma \in A$ is elliptic $s$-stable, and $Y=\bigcap_{\gamma \in A} \operatorname{Fix}(\gamma)$.

It is clear that the definition of an $s$-stable elliptic element does not depend on the choice of fixed point. Also, if $\gamma$ is an elliptic $s$-stable isometry then $\operatorname{Fix}(\gamma)=\operatorname{Fix}\left(\gamma^{i}\right)$, and for every elliptic isometry $\gamma^{\prime}, \gamma^{\prime}$ commutes with $\gamma$ iff it commutes with $\gamma^{i}$. This is easily seen by looking at a point fixed by both commuting isometries.

The notion of stability was originally introduced in [3, Section 12.4] for hyperbolic isometries. There, a hyperbolic element $\gamma$ is called $s$-stable if $\operatorname{Min}\left(\gamma^{i}\right)=\operatorname{Min}(\gamma)$ for $i=1, \ldots, s$ (there is no requirement that centralizers are equal). Although our requirement is stronger, we still have the following "stabilization" property which is established in [3, Section 12.5] for hyperbolic elements:

Proposition 2.5 For every $s \in \mathbb{N}$ there exists $s^{*}=s^{*}(s, n)$ such that for every isometry $\gamma$ of $X$ there is some $j \leq s^{*}$ such that $\gamma^{i}$ is $s$-stable.

Proof Clearly, it suffices to prove the analog statement for $g \in \mathrm{SO}(n)$. Let us view $\mathrm{SO}(n)$ as a group of matrices over $\mathbb{C}$. An element $h \in \mathrm{SO}(n)$ commutes with $g$ iff there is a basis whose vectors are eigenvectors of both $g$ and $h$.

It is easy to see that if all eigenvalues of $g$ have argument $<\pi / s$ then $i^{\text {th }}(i \leq s)$ powers of distinct eigenvalues remain distinct, thus every eigenvector of $g^{i}$ is an eigenvector of $g$. Hence, $g$ commutes with $h$ iff $g^{i}$ commutes with $h$. Also, under this assumption, the eigenspace of eigenvalue 1 is the same for $g$ and for $g^{i}$, and hence $\operatorname{Fix}(g)=\operatorname{Fix}\left(g^{i}\right)$. 
Now, partition $T=\{z \in \mathbb{C}:|z|=1\}$ into $2 s$ intervals $U_{k}=\left\{e^{\pi x / s}: k \leq x<k+1\right\}$ for $k=0, \ldots, 2 s-1$, and endow this to a partition of $T^{n}$ into $(2 s)^{n}$ parts. By a pigeonhole principal argument, if $\left(z_{1}, \ldots, z_{n}\right) \in T^{n}$ then there exists $1 \leq i \leq(2 s)^{n}+1$ such that $z_{j}^{i} \in U_{0}$ for all $1 \leq j \leq n$. We set $s^{*}=(2 s)^{n}+1$.

Considering $g \in \mathrm{SO}(n)$ again, we identify its eigenvalues as a tuple in $T^{n}$. Then there exists $1 \leq i \leq s^{*}$ such that all eigenvalues of $g$ have argument $<\pi / s$, and we are done.

\section{Bounding the thin part}

In the classical study of manifolds of non-positive curvature, it is usually beneficial to decompose a manifold into its "thick" and "thin" parts, ie, parts with injectivity radius bounded from below, and from above, respectively.

Roughly speaking, the topology of the thin part can be complicated and is somewhat controlled by the Margulis Lemma. When $\Gamma$ contains elliptic elements, the thin part contains all singular submanifolds, and its complexity is even greater. However, the Margulis Lemma can still be used to show that sufficiently stable singular submanifolds are more "well-behaved" than general singular submanifolds. The following two theorems make use of this general idea to make a quantitative statement.

With the exception of Theorem 3.5, the statements of this section hold whenever $X$ is a Hadamard manifold with sectional curvature normalized such that $-1 \leq K \leq 0$. We will not restate this assumption in any of the statements. Theorem 3.5 holds with the assumption that $-1 \leq K<0$.

We will make use of the following proposition, which is proved in [3, page 128].

Proposition 3.1 For every $\varepsilon>0$ and $k \in \mathbb{N}$ there is a $\delta>0$ such that if $\left|\Gamma_{\varepsilon}(x)\right| \leq k$ then there is a point $y \in X, d(x, y)<\varepsilon / 4$, and $d_{\Gamma}(y) \geq \delta$.

The following theorem is motivated by the analogous Theorem 12.11 of [3]. The proof of the latter deals with (stable) minimal translation sets of hyperbolic elements in non-positively curved manifolds, and overcomes difficulties that arise from the fact that they may have Euclidean factors. It relies on the assumption that the group acts freely. In our setting, the curvature is negative, however elliptic elements create a new difficulty. 
Theorem 3.2 Let $m=m_{n}$ be the constant of the Margulis Lemma, and let $\Sigma$ be a set of non-conjugate singular $m$-stable submanifolds in $X$. For every $\varepsilon_{1}>0$ there is an $\varepsilon_{2}=\varepsilon_{2}\left(\varepsilon_{1}, n\right)>0$ such that:

$$
\sum_{Y \in \Sigma} \varepsilon_{1}-\operatorname{ess}-\operatorname{vol}\left(Y / \Gamma_{Y}\right) \leq \varepsilon_{2}-\operatorname{ess}-\operatorname{vol}(X / \Gamma)
$$

Corollary 3.3 With the notation of the theorem, if $\varepsilon_{2}$-ess-vol $(X / \Gamma)$ is finite then there are only finitely many non-conjugate $m$-stable singular submanifolds with a non-empty $\varepsilon_{1}$-thick part.

We first prove:

Lemma 3.4 For $\delta>0$, there is some $\varepsilon>0$ such that the following holds: Let $Y \subseteq X$ be an $m$-stable singular submanifold $\left(m=m_{n}\right)$, and let $A \subset \Gamma$ be a set of elliptic elements such that $Y=\bigcap_{\alpha \in A} \operatorname{Fix}(\alpha)$. Let $x \in X$ be a point having the following properties:

(1) For every $\gamma \in \Gamma_{Y}$, if $d_{\gamma}(x)<\delta$ then $\gamma$ fixes $Y$ pointwise.

(2) $A \subseteq \Gamma_{\varepsilon}(x)$.

Then $\Gamma_{\varepsilon}(x)$ is finite, and $\bigcap_{\gamma \in \Gamma_{\varepsilon}(x)} \operatorname{Fix}(\gamma)=Y$.

Proof Let $k=k(\delta, n)$ be the constant introduced in the remark following the Margulis Lemma (Theorem 2.1). Take $\varepsilon=\min \left(\varepsilon_{n}, \delta /\left(2 m 4^{k}\right)\right)$. Let $N$ be the normal nilpotent subgroup of $\Gamma_{\varepsilon}(x)$ of index $i \leq m$.

We claim that for every $j$, there are generators of $N$ such that every $j$-fold iterated commutator $\gamma$ in those generators has $d_{\gamma}(x)<\delta$. Indeed, for $j \geq k$ take those "commutator contracted" generators used to define $k$. For $j<k$, by Lemma $2.3, N$ is generated by elements with $d_{\gamma}(x)<2 m \varepsilon$. Thus if $\gamma$ is a $j$-fold iterated commutator of these generators, then $d_{\gamma}(x)<2 m 4^{k} \varepsilon=\delta$.

We have $A \subset \Gamma_{\varepsilon}(x)$, and thus $B=\left\{\alpha^{i}: \alpha \in A\right\}$ is contained in $N$. Stability of the elements of $A$ implies that $Y=\bigcap_{\beta \in B} \operatorname{Fix}(\beta)$.

We claim that $N$ fixes $Y$ pointwise, and in particular, is finite. Denote by $N^{(j)}$ the $j^{\text {th }}$ term in the lower central series of $N$. The proof is by reverse induction. Suppose $N^{(j+1)}$ fixes $Y$. As we have noted, there are generators of $N$ such that if $\gamma$ is a $j$-fold iterated commutator of these generators then $d_{\gamma}(x)<\delta$. For each $\beta \in B$, $[\gamma, \beta] \in N^{(j+1)}$ fixes $Y$. It follows that $\gamma Y \subseteq \operatorname{Fix}(\beta)$. Therefore, $\gamma$ keeps $Y$ invariant. But since $d_{\gamma}(x)<\delta$, it follows by our assumptions that $\gamma$ fixes $Y$ pointwise. Finally, 
recall that $N^{(j)}$ is generated by $j$-fold iterated commutators of (any set of) generators of $N$ modulo $N^{(j+1)}$. It follows that $N^{(j)}$ fixes $Y$ pointwise.

Since $N$ fixes $Y$ pointwise and $B \subseteq N$, we have that $\bigcap_{\gamma \in N} \operatorname{Fix}(\gamma)=Y$. Normality of $N$ implies that $Y$ is $\Gamma_{\varepsilon}(x)$-invariant. It follows by the same argument as above that $\Gamma_{\varepsilon}(x)$ fixes $Y$ pointwise.

Remark The proof brings into mind an interesting question: Is there a uniform bound on the nilpotency degree of a discrete nilpotent group of isometries of $X$ ? This is easily seen to be true if $X$ is a symmetric space, in which case the group is linear. The answer is also positive if $\Gamma$ is torsion-free; this follows from Schroeder [18] together with Raghunathan [16, Lemma 2.19].

Before proving Theorem 3.2, let us explain the situation in which the previous lemma will be used. In the proof of the theorem, we will have a point $y \in Y$ which is in the $2 \delta-$ thick part of $Y / \Gamma_{Y}$, and a point $x \in X$ with $d\left(\pi_{Y}(x), y\right)<\delta / 2(x=y$ being a special case). Then $x$ satisfies the first assumption because if $d_{\gamma}(x)<\delta$ then $d_{\gamma}\left(\pi_{Y}(x)\right)<\delta$ and consequentially $d_{\gamma}(y)<2 \delta$. Our choice of $y$ implies that if $\gamma \in \Gamma_{Y}$ then $\gamma$ fixes $Y$ pointwise.

Proof of Theorem 3.2 Let us first note that it suffices to prove the theorem assuming $X \notin \Sigma$. Indeed, if $\varepsilon_{2}<\varepsilon_{1}$ is a constant for which (4) holds whenever $X \notin \Sigma$, then (4) holds for all sets $\Sigma$ with $\varepsilon_{2} / 2$ instead of $\varepsilon_{2}$, because

$$
\varepsilon_{1} \text {-ess-vol }\left(X / \Gamma_{X}\right)+\varepsilon_{2} \text {-ess-vol }(X / \Gamma) \leq \frac{\varepsilon_{2}}{2} \text {-ess-vol }(X / \Gamma) .
$$

We will also assume that $\Sigma$ is finite. Clearly, if (4) holds whenever $\Sigma$ is finite, it also holds when $\Sigma$ is infinite.

We proceed by induction on dimension. Suppose that the theorem holds for manifolds of dimension $<n$. Then for every $d<n$ there exists $\varepsilon_{2}^{(d)}>0$, such that (4) holds (with $\varepsilon_{2}^{(d)}$ for $\varepsilon_{2}$ ) for manifolds of dimension $d$. Take:

$$
\varepsilon_{3}=\min \left\{\varepsilon_{2}^{(d)}: d<n\right\}
$$

Clearly, (4) still holds if we replace $\varepsilon_{2}^{(d)}$ by $\varepsilon_{3}$.

Let $Z$ be an $m$-stable singular submanifold in $X$. Denote by $\Sigma_{Z} \subseteq \Sigma$ the set of $m$-stable singular submanifolds that are properly contained in $Z$. Suppose $Y \in \Sigma_{Z}$, and let $A_{Y}$ (resp. $A_{Z}$ ) be the set of all $m$-stable elliptic isometries fixing $Y$ (resp. $Z$ ) pointwise. Thus $\bigcap_{\gamma \in A_{Y}} \operatorname{Fix}(\gamma)=Y$ and $\bigcap_{\gamma \in A_{Z}} \operatorname{Fix}(\gamma)=Z$. Clearly, $A_{Z} \subsetneq A_{Y}$. Pick any $y \in Y$. Then $\Gamma_{y}$ has a normal abelian subgroup of index $i<m$ and clearly 
$A_{Y} \subseteq \Gamma_{y}$. We deduce that for every $\gamma_{1}, \gamma_{2} \in A_{Y}, \gamma_{1}^{i}, \gamma_{2}^{i}$ commute, and by the stability condition, $\gamma_{1}, \gamma_{2}$ commute. It follows that every element of $A_{Y}$ keeps $Z$ invariant. Thus, $Y$ can be considered an $m$-stable submanifold of $Z$, with respect to the group of isometries $\Gamma_{Z}$.

Furthermore, the Margulis constant $m_{n}$ is increasing with the dimension $n$. Hence $Y$ is also $m_{d}$-stable, $d=\operatorname{dim}(Z)$. By the induction hypothesis we have

$$
\sum_{Y \in \Sigma_{Z}} \varepsilon_{1} \text {-ess-vol }\left(Y / \Gamma_{Y, Z}\right) \leq \varepsilon_{3}-\text { ess-vol }\left(Z / \Gamma_{Z}\right)
$$

where $\Gamma_{Y, Z}$ is short for $\Gamma_{Y} \cap \Gamma_{Z}$.

Since our inequality involves $\varepsilon_{1}$-ess-vol $\left(Y / \Gamma_{Y}\right)$, we have to bound this number by $\varepsilon_{1}$-ess-vol $\left(Y / \Gamma_{Y, Z}\right)$. To this end, let $y_{1}, \ldots, y_{k}$ be points in $Y$ whose projection to $Y / \Gamma_{Y}$ is a $2 \varepsilon_{1}$-discrete set, and assume $d_{\Gamma_{Y}}\left(y_{i}\right)>2 \varepsilon_{1}$. Since $\Gamma_{Y, Z} \leq \Gamma_{Y}$, we have $d_{\Gamma_{Y, Z}}\left(y_{i}\right)>2 \varepsilon_{1}$. Also, the projection of these points to $Y / \Gamma_{Y, Z}(x)$ is $2 \varepsilon_{1}-$ discrete, because the group acting on $Y$ is smaller. Hence $\varepsilon_{1}$-ess-vol $\left(Y / \Gamma_{Y}\right) \leq$ $\varepsilon_{1}$-ess-vol $\left(Y / \Gamma_{Y, Z}\right)$.

Let $\Sigma^{\prime} \subseteq \Sigma$ be the set of $m$-stable submanifolds which are maximal in $\Sigma \backslash\{X\}$ with respect to inclusion. Then we have

$$
\begin{aligned}
\sum_{Y \in \Sigma \backslash\{X\}} \varepsilon_{1}-\mathrm{ess}-\operatorname{vol}\left(Y / \Gamma_{Y}\right) & \leq \sum_{Z \in \Sigma^{\prime}} \sum_{Z \supseteq Y \in \Sigma} \varepsilon_{1}-\operatorname{ess}-\operatorname{vol}\left(Y / \Gamma_{Y}\right) \\
& \leq \sum_{Z \in \Sigma^{\prime}} \sum_{Z \supseteq Y \in \Sigma} \varepsilon_{1}-\operatorname{ess}-\operatorname{vol}\left(Y / \Gamma_{Y, Z}\right) \\
& \leq \sum_{Z \in \Sigma^{\prime}} \varepsilon_{3}-\operatorname{ess}-\operatorname{vol}\left(Z / \Gamma_{Z}\right)
\end{aligned}
$$

and our problem is reduced to the case of maximal submanifolds.

We will henceforth assume $\Sigma$ is a set of maximal non-trivial $m$-stable submanifolds, and prove that there exists $\varepsilon_{2}$ for which (4) holds.

Here is the strategy of the proof: To a point on the $2 \varepsilon_{1}$-thick part of a stable singular submanifold we assign a ball of radius $\varepsilon_{2}$ in the $2 \varepsilon_{2}$-thick part of $X / \Gamma$. We then show that for a collection of points realizing the sum of $\varepsilon_{1}$-ess-vol of all relevant submanifolds, the assigned balls are pairwise disjoint in $X / \Gamma$.

Take $\varepsilon_{3}$ given by Lemma 3.4 for $\delta=2 \varepsilon_{1}$. Note that $\varepsilon_{3}<\varepsilon_{1} / 2$. Set $\varepsilon_{4}=\varepsilon_{3} /\left(4 m m^{*}\right)$, where $m^{*}$ is the constant of Proposition 2.5.

Let $Z \in \Sigma^{\prime}$, and let $z \in Z$ be a point whose image in $Z / \Gamma_{Z}$ is in the $2 \varepsilon_{1}$-thick part. Let $A_{Z}$ be the set of all $m$-stable elements fixing $Z$. Since $Z$ is not properly 
contained in any other $m$-stable singular submanifold, we have that $\operatorname{Fix}(\gamma)=Z$ for every $\gamma \in A_{Z}$. By Lemma 3.4, $\Gamma_{\varepsilon_{3}}(z)$ is finite and every $\gamma \in \Gamma_{\varepsilon_{3}}(z)$ fixes $Z$, and $z$ in particular.

Let $c:[0, \infty) \rightarrow X$ be a geodesic ray with $c(0)=z$ and $c^{\prime}(0) \perp Z$. For every $\gamma \in A_{Z}$, $d_{\gamma}$ is not constant along $c$, and by convexity it is hence strictly increasing. We fix $y=c\left(t_{0}\right), t_{0}>0$, such that $d_{\gamma}(y) \geq \varepsilon_{3} / 2$ for all $\gamma \in A_{Z}$, and $d_{\alpha}(y)=\varepsilon_{3} / 2$ for some $\alpha \in A_{Z}$.

Since $\alpha \in \Gamma_{\varepsilon_{3}}(y)$, and $\pi_{Z}(y)=z$, Lemma 3.4 applies again. We deduce that $\Gamma_{\varepsilon_{3}}(y)$ is finite, and its elements fix $Z$ pointwise. This is a fortiori true for $\Gamma_{\varepsilon_{4}}(y)$. Let $N$ be the normal abelian subgroup of $\Gamma_{\varepsilon_{4}}(y)$, of index $i \leq m$. Let $\gamma \in N$ with $d_{\gamma}(y)<2 m \varepsilon_{4}$ (recall that $N$ is generated by such elements). By Proposition 2.5, $\gamma^{j}$ is $m$-stable, for some $j \leq m^{*}$. Since $\operatorname{Fix}\left(\gamma^{j}\right) \supseteq Z$, and $Z$ is maximal, either $\gamma^{j}$ is trivial, or $\gamma^{j} \in A_{Z}$. But the latter is impossible, because $d_{\gamma^{j}}(y) \leq j \cdot d_{\gamma}(y)<m^{*} \cdot 2 m \varepsilon_{4}=\varepsilon_{3} / 2$. Therefore, $N$ is generated by elements of order $\leq m^{*}$. Since $N$ is abelian, every element of it has order $\leq m^{*}$ !. But $N$ is an abelian subgroup of $\operatorname{SO}(n)$, hence it is generated by at most $n$ elements (not necessarily those taken before), hence $|N| \leq\left(m^{*} !\right)^{n}$ and $\left|\Gamma_{\varepsilon_{4}}(y)\right| \leq m\left(m^{*} !\right)^{n}$.

Now, we fix a constant $\varepsilon_{2}$ by Proposition 3.1, such that there exists $x \in X$ with $d(x, y)<\varepsilon_{4} / 4$ and $d_{\Gamma}(x) \geq 2 \varepsilon_{2}$. We may assume $\varepsilon_{2} \leq \min \left(\varepsilon_{1} / 4, \varepsilon_{3} / 8\right)$. Note that $\varepsilon_{2}$ depends on $\varepsilon_{4}$ and on $m\left(m^{*} !\right)^{n}$, thus it depends only on $\varepsilon_{1}$ and $n$.

Let $B(z)$ be the ball of radius $\varepsilon_{2}$ centered at $x$. We claim that every point $w \in B(z)$ has the following properties:

(a) $d\left(z, \pi_{Z}(w)\right)<\varepsilon_{1}$, and

(b) $\Gamma_{\varepsilon_{3}}(w)$ is finite and $\bigcap_{\gamma \in \Gamma_{\varepsilon_{3}}(w)} \operatorname{Fix}(\gamma)=Z$.

Property (a) is immediate, because:

$$
d\left(z, \pi_{Z}(w)\right)=d\left(\pi_{Z}(y), \pi_{Z}(w)\right) \leq d(y, w) \leq \varepsilon_{2}+\varepsilon_{4} / 4<\varepsilon_{1}
$$

Since $d_{\alpha}(y)=\varepsilon_{3} / 2$, and $d(w, y) \leq \varepsilon_{2}+\varepsilon_{4} / 2$, we have:

$$
d_{\alpha}(w) \leq d_{\alpha}(y)+2 d(w, y)<\varepsilon_{3} / 2+2 \varepsilon_{2}+\varepsilon_{4}<\varepsilon_{3}
$$

Thus $\alpha \in \Gamma_{\varepsilon_{3}}(w)$, and in light of (a), it follows from Lemma 3.4 that property (b) holds.

To complete the proof, let $\Sigma^{\prime}=\left\{Z_{1}, \ldots, Z_{s}\right\}$ and for each $1 \leq i \leq s$ choose a set of points $\left\{z_{i, 1}, \ldots, z_{i, t_{i}}\right\}$ in $Z_{i}$ whose projections to $Z_{i} / \Gamma_{Z_{i}}$ are a $2 \varepsilon_{1}$-discrete set in the $2 \varepsilon_{1}$-thick part, and such that $t_{i}=\varepsilon_{1}$-ess-vol $\left(Z_{i} / \Gamma_{Z_{i}}\right)$. 
Consider the collection of balls $\left\{B\left(z_{i, j}\right): 1 \leq i \leq s, 1 \leq j \leq t_{i}\right\}$, and their projections $\widetilde{B}\left(z_{i, j}\right)$ to $X / \Gamma$. Recall that $B\left(z_{i, j}\right)$ is centered around a point in the $2 \varepsilon_{2}$-thick part of $X / \Gamma$. We claim that these balls are pairwise disjoint.

To this end, suppose $\widetilde{B}\left(z_{i, j}\right)$ and $\widetilde{B}\left(z_{i^{\prime}, j^{\prime}}\right)$ have non-empty intersection. Then there exists $w \in B\left(z_{i, j}\right)$ and $\gamma \in \Gamma$ such that $\gamma w \in B\left(z_{i^{\prime}, j^{\prime}}\right)$. By property (b), we deduce that $\gamma Z_{i^{\prime}}=Z_{i}$. By our assumption on $\Sigma$, we deduce that $i=i^{\prime}$ and $\gamma \in \Gamma_{Z_{i}}$. Now property (a) implies that the images of $z_{i, j}$ and $z_{i^{\prime}, j^{\prime}}$ in $Z_{i} / \Gamma_{Z_{i}}$ have distance $<2 \varepsilon_{1}$. Thus $z_{i, j}=z_{i^{\prime}, j^{\prime}}$.

A second type of minimal sets that arises in the thin part are "short geodesics". These are the images of axes of hyperbolic isometries in $\Gamma$ with translation length less than $\varepsilon_{n} / 2$ (the Margulis constant). In the torsion-free setting, bounding the number short geodesics is a straightforward consequence of the Margulis Lemma (eg, [3, Lemma 10.4]). The presence of finite order elements introduces new difficulties that are dealt with in the proof of the following theorem.

Theorem 3.5 Assume that $-1 \leq K<0$. Let $\varepsilon=\varepsilon_{n}$, and $\mathcal{C}$ be a set of non-conjugate geodesics in $X$, such that for each $c \in \mathcal{C}$ there exists a hyperbolic element $\alpha \in \Gamma$ with $C=\operatorname{Min}(\alpha)$, and $\min d_{\alpha}<\varepsilon / 2$. Then $|\mathcal{C}| \leq \mu$-ess-vol $(X / \Gamma)$, where $\mu$ is a constant depending on $n$.

Proof Let $C \in \mathcal{C}$, and let $\alpha$ be a hyperbolic element with axis $C$ and $\min d_{\alpha}<\varepsilon / 2$. Pick $x \in C$, and let $N$ be the nilpotent normal subgroup of $\Gamma_{\varepsilon}(x)$. Denote by $N_{s}$ the set of semisimple elements in $N$. Then, by Lemma 2.2, $K=\bigcap_{\gamma \in N_{s}} \operatorname{Min}(\gamma)$ is a non-empty convex and $N$-invariant. Now, some power of $\alpha$ is in $N$, hence in $N_{s}$. Therefore, $K$ is contained in $C$ and is $\alpha$-invariant. It follows that $K=C$. Thus $\Gamma_{\varepsilon}(x)$ keeps $C$ invariant.

Let $Y$ be an $m$-stable $\left(m=m_{n}\right)$ singular manifold containing $C$ that is minimal w.r.t. inclusion (this exists because $X$ itself is $m$-stable). Let $A_{Y}$ be the set of all elliptic $m$-stable elements fixing $Y$, and $A_{C}$ be the set of all elliptic $m$-stable elements fixing $C$. The minimality of $Y$ implies that for every $\gamma \in A_{C} \backslash A_{Y}$, Fix $(\gamma) \cap Y=C$. Also, let $H_{C}$ be the set of hyperbolic elements with axis $C$.

Take a geodesic ray $c:[0, \infty) \rightarrow Y$ with $c(0)=x$ and $c^{\prime}(0) \perp C$. If $\gamma \in A_{C} \backslash A_{Y}$ or $\gamma \in H_{C}$ then $\gamma$ keeps $C$ invariant, and $c$ is not contained in $\operatorname{Min}(\gamma)$. Thus, $d_{\gamma}$ is strictly increasing along $c$. Fix $y=c\left(t_{0}\right)$ such that $d_{\gamma}(y) \geq \varepsilon / 2$ for all $\gamma \in\left(A_{C} \backslash A_{Y}\right) \cup H_{C}$ and $d_{\alpha}(y)=\varepsilon / 2$ for some $\alpha \in\left(A_{C} \backslash A_{Y}\right) \cup H_{C}$. 
Claim Let $z \in Y$ and $d(z, y)<\varepsilon / 4$. If $\Gamma_{\varepsilon}(z)$ is infinite then it contains a hyperbolic element and every hyperbolic element $\gamma \in \Gamma_{\varepsilon}(z)$ has $\operatorname{Min}(\gamma)=C$. If $\Gamma_{\varepsilon}(z)$ is finite then $\bigcap_{\gamma \in \Gamma_{\varepsilon}(z)} \operatorname{Fix}(\gamma) \subseteq C$. In either case, $\Gamma_{\varepsilon}(z)$ keeps $C$ invariant.

Proof First note that $A_{Y} \subset \Gamma_{\varepsilon}(z)$ because $z \in Y$. Also, $\alpha \in \Gamma_{\varepsilon}(z)$ because $d_{\alpha}(y)<$ $\varepsilon / 2$ and $d(y, z)<\varepsilon / 4$. Let $N$ be the normal nilpotent subgroup of $\Gamma_{\varepsilon}(z)$ with index $i \leq m$.

Suppose $\Gamma_{\varepsilon}(z)$ is infinite. Then $N$ is a finitely generated infinite nilpotent group, and thus contains a central element $\beta$ of infinite order (cf [4]). For every $\gamma \in A_{Y} \cup\{\alpha\}$ we have $\gamma^{i} \in N$. Since $\beta$ commutes with $\gamma^{i}, \beta$ keeps $\operatorname{Min}\left(\gamma^{i}\right)=\operatorname{Min}(\gamma)$ invariant (note that the latter equation holds whether $\gamma=\alpha$ is hyperbolic or elliptic, in which case it is $m$-stable). Hence, $\beta$ keeps $C=Y \cap \operatorname{Min}(\alpha)$ invariant. Since $C$ is a line, $\beta$ is hyperbolic with axis $C$. It follows that $\Gamma_{\varepsilon}(z)$ keeps $C$ invariant, and that any hyperbolic element in $\Gamma_{\varepsilon}(z)$ has axis $C$.

Suppose now that $\Gamma_{\varepsilon}(z)$ is finite. In this case, $\alpha \in A_{C} \backslash A_{Y}$, because it is not hyperbolic. Since $A_{Y} \cup\{\alpha\} \subset \Gamma_{\varepsilon}(z)$, we have that $\bigcap_{\gamma \in \Gamma_{\varepsilon}(z)} \operatorname{Fix}(\gamma) \subseteq Y \cap \operatorname{Fix}(\alpha)=C$. To see that $\Gamma_{\varepsilon}(z)$ keeps $C$ invariant, pick $z^{\prime} \in \bigcap_{\gamma \in \Gamma_{\varepsilon}(z)} \operatorname{Fix}(\gamma)$. Then $\Gamma_{\varepsilon}(z) \leq \Gamma_{\varepsilon}\left(z^{\prime}\right)$. Since $\Gamma_{\varepsilon}\left(z^{\prime}\right)$ contains a hyperbolic element with axis $C$, all its elements keep $C$ invariant, and thus the same is true for $\Gamma_{\varepsilon}(z)$.

We return to the proof of the theorem. Let $\varepsilon_{1}=\varepsilon / 4 m^{*}$ ( $m^{*}$ is the constant of Proposition 2.5). By the claim, all elements of $\Gamma_{\varepsilon_{1}}(y)$ keep $C$ invariant. We prove that $\Gamma_{\varepsilon_{1}}(y)$ is finite. Suppose by contradiction that it is infinite. Then a fortiori $\Gamma_{\varepsilon}(y)$ is infinite. Now, by Lemma 2.3, $\Gamma_{\varepsilon_{1}}(y)$ contains an element $\gamma$ of infinite order and $d_{\gamma}(y)<2 m \varepsilon_{1}<\varepsilon / 2$. Since $\gamma$ keeps $C$ invariant, it must be hyperbolic with axis $C$. But this is impossible by the choice of $y$.

Let $N$ be the normal abelian subgroup of $\Gamma_{\varepsilon_{1}}(y) \cap \Gamma_{Y}$. Let $\gamma \in N$. Since $\gamma$ is elliptic and keeps the line $C$ invariant, $\gamma^{2}$ fixes $C$ pointwise. For some $j \leq m^{*}$, $\gamma^{2 j}$ is $m$-stable, thus $\gamma^{2 j} \in A_{C}$. But since $d_{\gamma^{2 j}}(y)<2 m^{*} \varepsilon_{1}=\varepsilon / 2$ we deduce that $\gamma^{2 j} \in A_{Y}$, hence $\gamma \in \Gamma_{Y}^{1}$. This means that every element in $N /\left(N \cap \Gamma_{Y}^{1}\right)$ has order $\leq 2 m^{*}$, and since this group is generated at most $n$ elements, $\left|N / N \cap \Gamma_{Y}^{1}\right| \leq\left(2 m^{*}\right)^{n}$, and $\left|\Gamma_{\varepsilon_{1}}(y) \cap \Gamma_{Y} / \Gamma_{Y}^{1}\right| \leq m\left(2 m^{*}\right)^{n}$.

We now apply Proposition 3.1 to $\Gamma_{\varepsilon_{1}}(y) \cap \Gamma_{Y} / \Gamma_{Y}^{1}$, regarding it as a subgroup of isometries of $Y$. There is a constant $\varepsilon_{2}>0$ and a point $z \in Y$ with $d(y, z)<\varepsilon_{1} / 4$ and $d_{\Gamma_{Y}}(z)>2 \varepsilon_{2}$. We denote by $B(C)$ the ball of radius $\varepsilon_{2}$ inside $Y$ centered at $z$.

Now suppose $C, C^{\prime} \in \mathcal{C}, C \neq C^{\prime}$. Then either $B(C), B\left(C^{\prime}\right)$ are contained in nonconjugate $m$-stable submanifolds, or we may assume they are contained in the same 
$m$-stable submanifold $Y$. In the latter case, their images in $Y / \Gamma_{Y}$ are disjoint. Indeed, if they are not, then we may assume (by conjugation with an element of $\Gamma_{Y}$ ) that $B(C)$ meets $B\left(C^{\prime}\right)$, and this easily leads to a contradiction by the above claim. The argument is similar to that of the proof of the previous theorem.

We thus have

$$
|\mathcal{C}| \leq \sum_{Y \in \Sigma} \varepsilon_{2}-\text { ess-vol }\left(Y / \Gamma_{Y}\right) \leq \mu-\text { ess-vol }(X / \Gamma)
$$

where $\Sigma$ is a set of non-conjugate $m$-stable submanifolds that contain the geodesics in $\mathcal{C}$, and $\mu$ is a constant obtained by Theorem 3.2.

\section{Bounding the quasi-thick part}

We now turn to study the "thick" part. The topology of the "thick" part of a manifold with finite volume can be understood, to some extent, by means of a good cover of balls whose number is bounded by the volume of the manifold. This is useful if the "thin" part can be analyzed separately. Yet, in the previous section we have restricted our attention to a certain part of the "thin" part, namely, the stable singular submanifolds. To compensate for this shortcoming, we need to extend the standard notion of the $\varepsilon$-thick part.

In this section, $X$ is an $n$-dimensional Hadamard manifold with non-positive sectional curvature normalized such that $-1 \leq K \leq 0$. As always, $\Gamma$ is a discrete subgroup of $\operatorname{Isom}(X)$. Here we denote by $O=X / \Gamma$ the quotient orbifold.

Definition 4.1 For $\varepsilon>0$ and $m \in \mathbb{N}$ we define:

$$
X_{\geq \varepsilon, m}=\left\{x \in X:\left|\Gamma_{\varepsilon}(x)\right| \leq m\right\}
$$

Since this set is $\Gamma$-invariant, we can define the $(\varepsilon, m)$-quasi-thick part of $O$ as $O_{\geq \varepsilon, m}=X_{\geq \varepsilon, m} / \Gamma$.

Our goal is to show that $(\varepsilon, m)$-quasi-thick part of $O$ can be covered by a collection of sets with contractible intersections, such that the volume controls the size of the covers and the degree of intersections. We remark that by "cover" we mean that the union of the sets contains the quasi-thick part, and may be strictly larger. In precise terms:

Theorem 4.2 There are positive constants $\delta$ and $r$ depending on $\varepsilon, n$ and $m$, such that $O_{\leq \varepsilon, m}$ admits a finite open cover $\mathcal{B}$ with the following properties:

(1) The elements of $\mathcal{B}$ are metric balls in $O$ of radius $\leq \varepsilon / 4$. 
(2) A non-empty intersection of balls in $\mathcal{B}$ is contractible.

(3) The cardinality of $\mathcal{B}$ is bounded from above by $\delta$-ess-vol $(O)$.

(4) Each ball in $\mathcal{B}$ intersects at most $r$ balls in $\mathcal{B}$.

Note that $\delta$ and $r$ depend on the dimension $n$. However, since any smaller $\delta$ and larger $r$ will do, we may well choose constants that are good for orbifolds of dimension $\leq n$ (for given $\varepsilon$ and $m$ ).

Corollary 4.3 If $\delta$-ess-vol $(O)<\infty$ then $O_{\geq \varepsilon, m}$ is compact.

To prove this theorem, we begin by studying the geometry of singular sets-sets of points fixed by finite subgroups of $\Gamma$.

\subsection{Singular sets}

We introduce some ad hoc notation for describing the singular sets of groups of isometries. For a finite group of isometries $G<\operatorname{Isom}(X)$ we denote $F(G)=\cap_{g \in G} \operatorname{Fix}(g)$. For a group of isometries $\Delta<\operatorname{Isom}(X)$ we define:

$$
\begin{aligned}
\Sigma(\Delta) & =\{F(G): G<\Delta \text { finite }\} \\
\Sigma_{i}(\Delta) & =\{Y \in \Sigma(\Delta): \operatorname{dim}(Y)=i\} \\
\Sigma_{<i}(\Delta) & =\{Y \in \Sigma(\Delta): \operatorname{dim}(Y)<i\}
\end{aligned}
$$

We set $S_{i}(\Delta)=\bigcup_{Y \in \Sigma_{i}} Y$ and similarly $S_{<i}(\Delta)$. We omit reference to the group $\Delta$ where there is no ambiguity. It should be emphasized that in these notations we do not exclude the trivial element, or the trivial group. Thus $X \in \Sigma(\Delta)$ for group $\Delta$.

Note that $\Sigma$ and $\Sigma_{<i}$ are all closed under intersection.

We study some basic properties of singular sets in the context of the $(\varepsilon, m)$-quasi-thick part. All constants stipulated by these proposition may depend on $n$ (the dimension of $X$ ) and $m$, but not on any particular group of isometries. We also assume all " $\varepsilon$ " constants are smaller than half of the constant of the Margulis Lemma.

Proposition 4.4 Let $Y_{1}, Y_{2} \in \Sigma(\Gamma)$, and $y_{j} \in Y_{j} \cap X_{\geq \varepsilon, m}(j=1,2)$. If $d\left(y_{1}, y_{2}\right)<$ $\varepsilon / 2$ then there exists $G<\Gamma,|G| \leq m$ such that $Y_{1}, Y_{2} \in \Sigma(G)$ (in particular, $\left.Y_{1} \cap Y_{2} \neq \varnothing\right)$.

Proof Take $G=\Gamma_{\varepsilon}\left(y_{2}\right)$. Trivially, $\Gamma_{y_{2}}<G$ and by the hypothesis, also $\Gamma_{y_{1}}<G$. Since $Y_{j} \in \Sigma(\Gamma)$, we have that $F\left(\Gamma_{Y_{j}}^{1}\right)=Y_{j}$, and as $\Gamma_{Y_{j}}^{1} \leq \Gamma_{y_{j}} \leq G$, it follows that $Y_{j} \in \Sigma(G)$. 
Proposition 4.5 For $\varepsilon_{1}>0$ there exists $\varepsilon_{2}=\varepsilon_{2}\left(\varepsilon_{1}\right)>0$ so that the following holds: Let $G<\operatorname{Isom}(X)$ be a finite group, $|G| \leq m$, and let $Y_{1}, Y_{2} \in \Sigma(G)$ such that $Y_{1} \nsubseteq Y_{2}$ and $Y_{2} \nsubseteq Y_{1}$. If $y_{1} \in Y_{1}, y_{2} \in Y_{2}$ and $d\left(y_{1}, y_{2}\right)<\varepsilon_{2}$ then $d\left(y_{i}, Y_{1} \cap Y_{2}\right)<\varepsilon_{1}$ for $i=1,2$.

Proof Suppose that $d\left(y_{1}, Y_{1} \cap Y_{2}\right) \geq \varepsilon_{1}$. We will prove that there exists $\varepsilon_{2}=\varepsilon_{2}\left(\varepsilon_{1}\right)$ such that $d\left(y_{1}, y_{2}\right) \geq \varepsilon_{2}$.

Let $x$ be the projection of $y_{1}$ onto $Y_{1} \cap Y_{2}$. By our assumption, $d\left(y_{1}, x\right) \geq \varepsilon_{1}$, and we may proceed with the assumption that $x \neq y_{2}$. Consider the geodesic triangle with vertices $x, y_{1}, y_{2}$, and let $\alpha$ be the angle at $x$. By replacing $y_{2}$ with a point in $Y_{2}$ which is closer to $y_{1}$ (eg the projection of $y_{1}$ to $Y_{2}$ ), we may assume $\alpha \leq \pi / 2$. By comparison to a Euclidean triangle with sides of length $d\left(x, y_{1}\right), d\left(x, y_{2}\right)$ with an angle $\alpha$ between them, we deduce that $d\left(y_{1}, y_{2}\right) \geq \varepsilon_{1} \sin \alpha$. Our goal is to show that the right-hand side can be replaced by a function of $\varepsilon_{1}$ that does not depend on $\alpha$.

Let $\mathcal{Y}_{1}, \mathcal{Y}_{2} \subset T_{x}(X)$ be the tangent subspaces of $Y_{1}, Y_{2}$, respectively. Let $a$ be the geodesic segment $\left[x, y_{1}\right]$, and $b$ be the geodesic segment $\left[x, y_{2}\right]$, and let $A, B$ be the (unit) tangent vectors at $x$ of $a, b$, respectively. Since $x$ is the projection of $y_{1}$ to $Y_{1} \cap Y_{2}, a$ is perpendicular to $Y_{1} \cap Y_{2}$, and therefore $A$ is orthogonal to $\mathcal{Y}_{1} \cap \mathcal{Y}_{2}$. But since $B \in \mathcal{Y}_{2}$, the inner product of $A$ and $B$ is bounded away from 1 . Hence, the angle $\alpha$ is bounded from below by a constant $\alpha_{0}=\alpha_{0}\left(Y_{1}, Y_{2}\right)$.

By definition of $\Sigma(G)$, there are subgroups $H_{1}, H_{2}<G$ such that $Y_{i}=F\left(H_{i}\right)$, $i=1$, 2. Since both $H_{1}$ and $H_{2}$ fix $x$, we may identify them as subgroups of $\operatorname{SO}(n)$ via their induced action on the tangent plane $T_{x}(X)$. Since $\alpha_{0}\left(Y_{1}, Y_{2}\right)$ was determined by the action on the tangent space, it actually depends only on the embedding of $H_{1}, H_{2}$ in $\operatorname{SO}(n)$. Thus, we may write $\alpha_{0}\left(H_{1}, H_{2}\right)$ for $\alpha_{0}\left(Y_{1}, Y_{2}\right)$. Moreover, $\alpha_{0}\left(H_{1}, H_{2}\right)$ is invariant under (simultaneous) conjugation of $H_{1}$ and $H_{2}$ in $\mathrm{SO}(n)$.

Observe that, up to conjugation, there are finitely many pairs of subgroups $H_{1}, H_{2}$ in $\mathrm{SO}(n)$ that generate a group of size $\leq m$. Therefore, we may fix $\alpha_{1}>0$ to be the minimum of $\alpha_{0}\left(H_{1}, H_{2}\right)$ over all possible pairs $H_{1}, H_{2}<\mathrm{SO}(n)$ with $F\left(H_{1}\right) \nsubseteq F\left(H_{2}\right)$ and $F\left(H_{2}\right) \nsubseteq F\left(H_{1}\right)$ (here, $F\left(H_{i}\right)$ is the fixed set w.r.t. the linear action). By setting $\varepsilon_{2}=\varepsilon_{1} \sin \alpha_{1}$, we are done.

Let us state explicitly an immediate consequence. In what follows, we adapt the convention that $d(x, \varnothing)=\infty$, thus $d\left(x, S_{<0}(\Gamma)\right)>c$ for every $c>0$. Hence, the following propositions are meaningful when $i=0$, and in this case assumptions such as $d\left(x, S_{<i}(\Gamma)\right)>\varepsilon_{1}$ are superfluous. 
Proposition 4.6 Let $\varepsilon_{2}=\varepsilon_{2}\left(\varepsilon_{1}\right)$ as in Proposition 4.5. Let $Y_{1}, Y_{2} \in \Sigma(\Gamma)$, with $i=$ $\operatorname{dim}\left(Y_{2}\right) \leq \operatorname{dim}\left(Y_{1}\right)$. Let $y_{j} \in Y_{j} \cap X_{\geq \varepsilon, m}, j=1,2$. Suppose that $d\left(y_{1}, S_{<i}(\Gamma)\right) \geq \varepsilon_{1}$ or $d\left(y_{2}, S_{<i}(\Gamma)\right) \geq \varepsilon_{1}$. If $d\left(y_{1}, y_{2}\right)<\varepsilon_{2}$ then $Y_{2} \subseteq Y_{1}$.

Proof Suppose contrarily that $Y_{2} \nsubseteq Y_{1}$. Then by considering dimensions, also $Y_{1} \nsubseteq Y_{2}$. Since $d\left(y_{1}, y_{2}\right)<\varepsilon_{2}<\varepsilon / 2$, there is, by Proposition 4.4, some finite subgroup $G<\Gamma$ such that $Y_{1}, Y_{2} \in \Sigma(G)$, with $|G| \leq m$. Therefore, $Y_{1} \cap Y_{2}$ is non-empty, and by Proposition 4.5, we have that $d\left(y_{i}, Y_{1} \cap Y_{2}\right)<\varepsilon_{1}$ for both $i=1$ and $i=2$. But this contradicts our assumption, since $Y_{1} \cap Y_{2} \subseteq S_{<i}(\Gamma)$.

Proposition 4.7 Let $Y \in \Sigma(\Gamma), y \in Y \cap X_{\geq \varepsilon, m}$, and $i=\operatorname{dim}(Y)$. There exists $\varepsilon_{3}=\varepsilon_{3}\left(\varepsilon_{1}\right)$ such that if $d\left(y, S_{<i}(\Gamma)\right)>\varepsilon_{1}$ then every element of $\Gamma_{\varepsilon_{3}}(y)$ fixes $Y$ pointwise.

Proof Take $\varepsilon_{2}=\varepsilon_{2}\left(\varepsilon_{1}\right)$ given in Proposition 4.5; we may assume $\varepsilon_{2}<\varepsilon_{1}$. Fix $\varepsilon_{3}=2 \sin (\pi / m) \varepsilon_{2}$. Note that $\varepsilon_{3}<\varepsilon$, hence $G=\Gamma_{\varepsilon_{3}}(y)$ is a finite group of order $\leq m$, by the hypothesis.

Assume by contradiction that there are elements in $G$ that do not fix $Y$ pointwise. Then certainly there is such element $g \in G$ with $d_{g}(y)<\varepsilon_{3}$. First, we claim that $d(y, \operatorname{Fix}(g)) \geq \varepsilon_{2}$. We distinguish between two cases:

(1) $\operatorname{Fix}(g) \subsetneq Y$. Then $\operatorname{Fix}(g) \in \Sigma_{<i}(\Gamma)$ and this claim follows from the assumption $d\left(y, S_{<i}(\Gamma)\right)>\varepsilon_{1}$.

(2) $\operatorname{Fix}(g) \nsubseteq Y$. Suppose that $d(y, \operatorname{Fix}(g))<\varepsilon_{2}$. Take $y^{\prime} \in \operatorname{Fix}(g)$ with $d\left(y, y^{\prime}\right)=$ $d(y, \operatorname{Fix}(g))$. Applying Proposition 4.5 to the group $G$ and the sets $Y$ and $\operatorname{Fix}(g)$, we derive that $d(y, Y \cap \operatorname{Fix}(g))<\varepsilon_{1}$, a contradiction to our assumption that $d\left(y, S_{<i}(\Gamma)\right)>\varepsilon_{1}$.

Now, combining the facts that the order of $g$ is $\leq m$ and that $d(y, \operatorname{Fix}(g)) \geq \varepsilon_{2}$ yields that $d_{g}(y) \geq 2 \sin (\pi / m) \varepsilon_{2}=\varepsilon_{3}$ (cf [3, Section 12.2]), a contradiction.

\subsection{Foldable sets}

Definition 4.8 Let $U \subset X$ be a an open set, and $Y \subset X$ be a totally geodesic submanifold. We say $U$ is $Y$-foldable if it has the following properties:

- $U$ is convex and precisely invariant, ie, for every $\gamma \in \Gamma$ if $\gamma U \cap U \neq \varnothing$ then $\gamma U=U$.

- $\quad Y$ is fixed by $\Gamma_{U}$. 
- $\pi_{Y}(U) \subseteq U, \pi_{Y}$ being the projection to the closest point in $Y$.

- The image of $U \cap Y$ in $X / \Gamma$ is convex.

If $U$ is $Y$-foldable for some $Y$, we say it is foldable. If $U$ is $Y$-foldable, we say its image $\pi(U)$ is a folded set.

Remark If $U$ is $Y$-foldable then $U \cap Y$ injects to $X / \Gamma$. This follows from the assumption that $Y$ is fixed by $\Gamma_{U}$.

The notable property of folded sets is:

Proposition 4.9 Folded sets are contractible.

Proof Let $U \subset X$ be a foldable set. Since $U$ is convex, and $\pi_{Y}(U) \subseteq U$, there is a deformation retract of $U$ to $U \cap Y$, defined by the flow along the geodesics of the projection to $Y$. Since $\Gamma_{U}$ fixes $Y$, this retraction can be taken to be $\Gamma_{U}$-equivariant, and as $U$ is precisely invariant, it induces a retraction of $\pi(U)$ to $\pi(U \cap Y)$, which in turn is contractible.

Foldable sets appear naturally in certain neighborhoods of singular submanifolds. Let $Y \in \Sigma_{i}(i \leq n)$ and $y \in Y \backslash S_{<i}$. Observe that this implies that $\Gamma_{y}$ fixes $Y$. Indeed, since $Y \in \Sigma$, we have that $Y=F\left(\Gamma_{Y}^{1}\right)$. Now, $\Gamma_{Y}^{1} \leq \Gamma_{y}$, and had this inclusion been proper, $F\left(\Gamma_{y}\right)$ would have dimension less than $i$, contradicting the choice of $y$. Thus, $\Gamma_{Y}^{1}=\Gamma_{y}$.

Choose $\mu$ sufficiently small so that $\Gamma_{4 \mu}(y)$ fixes $Y$. Let $U=B(y, \mu)$. Then $U$ is convex and open. It is precisely invariant, because if $\gamma U$ intersects $U$ then $d_{\gamma}(y)<2 \mu$, and thus $\gamma y=y$ and $\gamma U=U$. It remains to check whether $U \cap Y$ injects to a convex

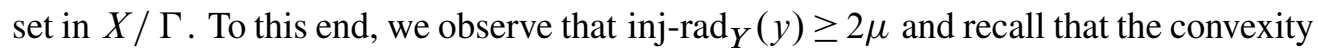
radius is half of the injectivity radius. All foldable balls mentioned henceforth are assumed to be constructed in this manner.

We will also want to consider intersections of such foldable balls. Let $B_{1}$ be a $Y-$ foldable ball centered at a point in $Y$. Suppose that $B_{2}, \ldots, B_{k}$ are foldable balls centered at points in $Y$, and that $U=\bigcap B_{i} \neq \varnothing$. Note that we do not require these balls to be $Y$-foldable. The intersection of precisely invariant sets is itself precisely invariant. Moreover, $\Gamma_{U}$ is a subgroup of $\Gamma_{B_{1}}$, so it fixes $Y$. For every $x \in U$, $\pi_{Y}(x) \in U$, because the projection to $Y$ does not increase distances to the centers of the balls (which are in $Y$ ). Now, $U \cap Y$ is convex because all balls are centered at points of $Y$. Since the image of $U \cap Y$ in $X / \Gamma$ is contained in the convex image of $B_{1} \cap Y$, it follows that the image of $U \cap Y$ is convex. This shows that $U$ is $Y$-foldable. 
Moreover, we claim that $\pi(U)=\bigcap \pi\left(B_{i}\right)$, hence the intersection of these folded sets is itself folded and thus contractible. This is corollary of the following proposition.

Proposition 4.10 Let $x_{1}, \ldots, x_{r} \in X$ and $\mu_{1}, \ldots, \mu_{r}>0$, such that $\Gamma_{4 \mu_{i}}\left(x_{i}\right)$ fixes $x_{i}$ for all $i$. Suppose the intersection of the balls $B_{i}=B\left(x_{i}, \mu_{i}\right)$ is non-empty. Then $\pi\left(\bigcap B_{i}\right)=\bigcap \pi\left(B_{i}\right)$.

Proof The preimage of $\bigcap \pi\left(B_{i}\right)$ is the union of intersections of the form $\bigcap \gamma_{i} B_{i}$, $\gamma_{i} \in \Gamma$. It therefore suffices to show that any such non-empty intersection is a translate of $\bigcap B_{i}$ by an element of $\Gamma$.

Let us assume $\mu_{1} \leq \mu_{i}$ for all $i>1$. Also, by translating the intersection with $\gamma_{1}^{-1}$, we may assume $\gamma_{1}=1$.

For every $i \geq 2, B_{1}$ intersects $\gamma_{i} B_{i}$. Thus $d\left(\gamma_{i} x_{i}, x_{1}\right)<\mu_{1}+\mu_{i} \leq 2 \mu_{i}$. Since also $d\left(x_{i}, x_{1}\right)<2 \mu_{i}$ we obtain $d_{\gamma_{i}}\left(x_{i}\right)<4 \mu_{i}$. By our assumption $\gamma_{i}$ fixes $x_{i}$ and thus leaves $B_{i}$ invariant. Thus $\bigcap \gamma_{i} B_{i}=\bigcap B_{i}$.

\section{Proof of Theorem 4.2}

Our cover of the $(\varepsilon, m)$-quasi-thick part will consist of folded balls centered at maximal discrete subsets of the $(\varepsilon / 2, m)$-quasi-thick part. We denote $O^{\prime}=O_{\geq \varepsilon / 2, m}$.

We fix a sequence $\mu_{0}>\mu_{1}>\cdots>\mu_{n}$ by taking $\mu_{-1}=\varepsilon / 2$ and

$$
\mu_{i+1}=\min \left(\frac{1}{6} \varepsilon_{2}\left(\mu_{i}\right), \frac{1}{12} \varepsilon_{3}\left(\mu_{i}\right), \frac{1}{2} \mu_{i}\right),
$$

$\varepsilon_{2}$ and $\varepsilon_{3}$ being the constants stipulated in Propositions 4.6 and 4.7, respectively.

Let $\mathcal{D}_{0}$ be a maximal $\mu_{0}$-discrete set in $O^{\prime} \cap \pi\left(S_{0}\right)$. For every $i>0$, let $\mathcal{D}_{i}$ be a maximal $\mu_{i}$-discrete set in $O^{\prime} \cap \pi\left(S_{i}\right) \backslash \bigcup_{j<i}\left(\pi\left(S_{j}\right)\right)_{\mu_{j}}$ (inductively). Here, $\left(\pi\left(S_{j}\right)\right)_{\mu_{j}}$ denotes the set of points with distance $<\mu_{j}$ of $\pi\left(S_{j}\right)$. Take $\mathcal{D}=\bigcup \mathcal{D}_{i}$. We claim that the collection of balls $\mathcal{B}=\left\{B\left(x, 3 \mu_{i}\right): x \in \mathcal{D}_{i}\right\}$ has the desired properties. This is proved in the following series of claims:

(1) The balls in $\mathcal{B}$ cover $O_{\geq \varepsilon, m}$, and their centers constitute a $\mu_{n}$-discrete set.

The discreteness is obvious. We show that the balls cover $O_{\geq \varepsilon, m}$.

First, let us note that if $x \in O_{\geq \varepsilon, m}$ and $d(x, y)<\varepsilon / 4$ then $y \in O^{\prime}$. Indeed, choose preimages $\tilde{x}, \tilde{y} \in X$ such that $d(\tilde{x}, \tilde{y})<\varepsilon / 4$. Then $\Gamma_{\varepsilon / 2}(\tilde{y}) \leq \Gamma_{\varepsilon}(\tilde{x})$, whence we deduce $\tilde{y} \in X_{\geq \varepsilon / 2, m}$ and $y \in O^{\prime}$.

Let $x \in O_{\geq \varepsilon, m}$. Take minimal $i$ such that $d\left(x, \pi\left(S_{i}\right)\right)<2 \mu_{i}$ (such $i$ exists because $\left.x \in \pi\left(S_{n}\right)\right)$. Now pick $y \in \pi\left(S_{i}\right)$ such that $d(x, y)<2 \mu_{i}$. 
We claim that $y \in \pi\left(S_{i}\right) \backslash \bigcup_{k<i}\left(\pi\left(S_{k}\right)\right)_{\mu_{k}}$. Indeed, if there is some $k<i$ and $z \in \pi\left(S_{k}\right)$ with $d(y, z)<\mu_{k}$ then $d(x, z)<2 \mu_{i}+\mu_{k} \leq 2 \mu_{k}$, contradicting the minimality of $i$. Now, since $d(x, y)<2 \mu_{i} \leq \varepsilon / 4$, we have noted that $y \in O^{\prime}$. Therefore, there is some $z \in \mathcal{D}_{i}$ such that $d(y, z)<\mu_{i}$. Hence $x \in B\left(z, 3 \mu_{i}\right)$.

(2) Each ball in $\mathcal{B}$ is folded; it is the image of a foldable ball.

Let $x \in \mathcal{D}_{i}$, and choose a preimage $\tilde{x} \in X_{\geq \varepsilon / 2, m}$ of $x$. Let $Y \in \Sigma_{i}$ be the singular submanifold containing $\tilde{x}$. As $d\left(x, \pi\left(S_{<i}\right)\right)>\mu_{i-1}$, also $d\left(\tilde{x}, S_{<i}\right)>\mu_{i-1}$. Bearing in mind that $12 \mu_{i} \leq \varepsilon_{3}\left(\mu_{i-1}\right)$, we deduce by Proposition 4.7 that every element of $\Gamma_{12 \mu_{i}}(\tilde{x})$ fixes $Y$ pointwise. Thus the ball $B\left(\tilde{x}, 3 \mu_{i}\right)$ is $Y$-foldable, and its image $B\left(x, 3 \mu_{i}\right)$ is folded.

(3) A non-empty intersection of balls in $\mathcal{B}$ is folded, and thus contractible.

Let $x_{1}, \ldots, x_{k} \in \mathcal{D}$, such that $x_{i} \in \mathcal{D}_{n_{i}}$ and suppose that the balls $B\left(x_{i}, 3 \mu_{n_{i}}\right)$ have a non-empty intersection. We may assume $n_{1}=\max \left\{n_{j}\right\}$.

Choose lifts $\tilde{x}_{1}, \ldots, \tilde{x}_{k} \in X_{\geq \varepsilon / 2, m}$ such that $d\left(\tilde{x}_{1}, \tilde{x}_{j}\right)<6 \mu_{n_{j}}$, and let $Y_{j} \in \Sigma_{n_{j}}$ the singular submanifold containing $\tilde{x}_{j}$.

Fix $j \geq 2$. Then $d\left(\tilde{x}_{1}, S_{<n_{j}}\right) \geq \mu_{n_{j}-1}$ and $d\left(\tilde{x}_{j}, S_{<n_{j}}\right) \geq \mu_{n_{j}-1}$. Since $d\left(\tilde{x}_{1}, \tilde{x}_{j}\right)<$ $6 \mu_{n_{j}} \leq \varepsilon_{2}\left(\mu_{j-1}\right)$, we may apply Proposition 4.6, and deduce that $Y_{j} \subseteq Y_{1}$ and hence $x_{j} \in Y_{1}$.

The results discussed before this proof all apply to this situation. Namely, the intersection of the balls $B\left(\tilde{x}_{i}, 3 \mu_{n_{i}}\right)$ is $Y_{1}$-foldable, because they are all centered in points on $Y_{1}$, and $B\left(\tilde{x}_{1}, 3 \mu_{n_{1}}\right)$ is $Y_{1}$-foldable. Also, the intersection of the folded balls $B\left(x_{i}, 3 \mu_{n_{1}}\right)$ is folded, and thus contractible.

(4) The cardinality of $\mathcal{B}$ is bounded by $\delta$-ess-vol, where $\delta=\delta(\varepsilon, m, n)$.

By Proposition 3.1, there exists $\delta$ such that if $\left|\Gamma_{\mu_{n}}(x)\right| \leq m$ then there exists a point $y$ with $d(x, y)<\mu_{n} / 4$ and $d_{\Gamma}(y) \geq 2 \delta$. Note that $\mu_{n}$ depends only on $\varepsilon, m$ and $n$, hence $\delta$ depends only on those constants. We may assume $\delta \leq 4 \mu_{n}$.

Let $x \in \mathcal{D}_{i}$ and let $\tilde{x} \in X$ be a lift. Since $\tilde{x} \in O^{\prime}$, we have that $\left|\Gamma_{\mu_{n}}(\tilde{x})\right| \leq\left|\Gamma_{\mu_{i}}(\tilde{x})\right| \leq m$. Thus there exists $\tilde{y}, d(\tilde{y}, \tilde{x})<\mu_{n} / 4$ and $d_{\Gamma}(\tilde{y}) \geq 2 \delta$. The projection of $\tilde{y}$ is a point in the $\delta$-thick part of $O$, which we denote $y_{x}$.

Since the set $\mathcal{D}$ is $\mu_{n}$-discrete, if $x, x^{\prime} \in \mathcal{D}$ then the corresponding points $y_{x}, y_{x^{\prime}}$ have $d\left(y_{x}, y_{x^{\prime}}\right) \geq \mu_{n} / 2 \geq 2 \delta$. The set $\left\{y_{x}: x \in \mathcal{D}\right\}$ is therefore $2 \delta$-discrete in the $\delta$-thick part. We deduce that $|\mathcal{B}|=|\mathcal{D}| \leq \delta$-ess-vol $(O)$.

(5) Each ball of $\mathcal{B}$ intersects at most $r$ balls, where $r=r(\varepsilon, m, n)$. 
Let $x \in \mathcal{D}_{i}$, and suppose $B\left(x, 3 \mu_{i}\right)$ intersects the balls $B\left(y_{j}, 3 \mu_{i_{j}}\right), j=1, \ldots, k$. Lift the center points to $\tilde{x}, \tilde{y}_{j}$ such that $d\left(\tilde{x}, \tilde{y}_{j}\right) \leq 6 \mu_{0}$. The ball $B\left(\tilde{x}, 9 \mu_{0}\right)$ contains the pairwise disjoint balls $B\left(\tilde{y}_{j}, \mu_{n} / 2\right)$. Thus the existence of the constant $r$ follows from a standard volume estimate.

We conclude this section with a proposition about homology of a (orbifold) fibration over the cover given by Theorem 4.2 (for definitions, see [20, page 318]). The precedent to this proposition is Lemma 12.12 in [3], that states the analogous result for a cover, rather than a fibration over it.

Proposition 4.11 Let $\mathcal{B}$ be the cover of $O_{\geq \varepsilon, m}$ obtained in Theorem 4.2, and let $r$ and $\delta$ be the constants defined there. Let $U=\bigcup_{B \in \mathcal{B}} B$, and let $E$ be a fibration over $U$ with fiber $F$ being a sphere or vector space. There is a function $h(r)$ (depending only on $r$ ), such that for coefficients in an integral domain $A$ of characteristic 0 :

$$
\mathrm{rk}_{A} H_{j}(E, A) \leq h(r) \cdot|\mathcal{B}| \leq h(r) \cdot \delta \text {-ess-vol }(O)
$$

Proof Let $V$ be a non-empty intersection of balls in $\mathcal{B}$. Recall that, by the proof of Theorem 4.2, $V$ is folded; moreover, it is a quotient of a contractible set in $X$ by a finite group of order $\leq m$. Let $\tilde{V} \subset X$ be a precisely invariant contractible set such that $V=\tilde{V} / \Gamma_{\tilde{V}}$, and $\left|\Gamma_{\tilde{V}}\right| \leq m$.

By the definition of a fibration over $U$, there is a fiber bundle $\left.\widetilde{E}\right|_{\widetilde{V}}$ with fiber $F$ over $\widetilde{V}$, and an action of $\Gamma_{\widetilde{V}}$ on $\widetilde{E}_{\widetilde{V}}$ which is compatible with the action on $\widetilde{V}$, such that $\left.\left.E\right|_{V} \simeq \widetilde{E}\right|_{\tilde{V}} / \Gamma_{\tilde{V}}$

Since $\tilde{V}$ is contractible, $\left.\widetilde{E}\right|_{\tilde{V}}$ is trivial (cf [19]). Moreover, by the proof of Proposition 4.9 , there is a $\Gamma_{\tilde{V}}$-invariant contraction of $\tilde{V}$ to a fixed point of $\Gamma_{\tilde{V}}$. Hence, the deformation retract of $\left.\widetilde{E}\right|_{\tilde{V}}$ to $F$ induces a deformation retract of $\left.E\right|_{V}$ to $F / \Gamma_{\tilde{V}}$, with the action given by the action of the group on the fiber over a fixed point in $\tilde{V}$.

If $F$ is a vector space, we conclude that $E \mid V$ is contractible. If $F$ is a sphere, we will resort to the following claim, which is a consequence of the transfer homomorphism [7]:

Claim If $G$ is a finite group acting on $F$ then:

$$
\text { rk } H_{j}(F / G, A) \leq \operatorname{rk} H_{j}(F, A)
$$

Either way, we conclude that $\operatorname{rk} H_{j}\left(\left.E\right|_{V}\right) \leq 1$. Thus, $E$ is a union of $k$ open sets, such that each set intersects at most $r$ other sets, and every intersection has a homology of rank bounded by 1 . 
The existence (and explicit calculation) of the function $h(r)$ now follows as in the proof of Lemma 12.12 in [3]; it is assumed there that a non-empty intersection of sets is diffeomorphic to $\mathbb{R}^{n}$, but this is used only to imply that the rank of homology is bounded by 1 .

Remark As noted in the introduction, this proposition is the only place throughout this work where the assumption on characteristic is required. If the coefficients are in, say, $\mathbb{F}_{p}$, then the claim cited in the proof is not valid. Nonetheless, the claim still holds if the order of the finite group is prime to $p$. It follows at once that the proposition holds whenever the orders of the finite subgroups of $\Gamma$ are all prime to $p$.

In the general setting, we may still hope for a uniform bound (ie, independent of $G$ ) on the homology of the quotient in lieu of (5). This leads to the question posed in the introduction, asking whether there exists a constant $D$ depending on $k$ (and possibly on $p$ ) such that for every finite group $G$ acting linearly on a sphere $S^{k}$, and for every $i$, we have $b_{i}\left(S^{k} / G, \mathbb{F}_{p}\right) \leq D$.

The fibrations to which this proposition is applied (p. 1145) arise from the action of $\Gamma$ on the tangent space of $X$. The fibers we are considering are vector spaces and spheres of dimension $\leq n-1$, and the action of the finite group on these fibers can be assumed to be linear. Therefore, an affirmative answer to the question above for $k \leq n-1$ would suffice to prove Theorem 1.1 for spaces of dimension $\leq n$.

\section{Betti numbers}

We can now prove Theorem 1.1. We will prove the following detailed version:

Theorem 5.1 Let $X$ be an $n$-dimensional Hadamard manifold with negative sectional curvature normalized such that $-1 \leq K<0$. Let $\Gamma$ be a discrete subgroup of $\operatorname{Isom}(X)$, such that $\varepsilon-\operatorname{ess}-\operatorname{vol}(X / \Gamma)$ is finite for every $\varepsilon>0$. There exists $v=v(n)>0$ such that:

$$
\sum_{i} \operatorname{rk} H_{i}(X / \Gamma, A) \leq v \text {-ess-vol }(X / \Gamma)
$$

where the coefficient ring $A$ is an integral domain of characteristic 0 .

For orbifolds of finite volume, it follows that

$$
\sum_{i} \operatorname{rk} H_{i}(X / \Gamma, A) \leq C(n) \cdot \operatorname{vol}(X / \Gamma) .
$$


The proof follows closely the steps of Gromov in [3, Section 13]. Namely, homology is bounded by using an intricately defined Morse function, studying its critical points, defining deformation retracts between its sublevel sets and relying on bounds of the type of Theorem 3.2. Some arguments are identical to those of Gromov, and are brought here in full for completeness. Others are streamlined for our setting. This is true, in particular, for Claims 2-5 and for arguments concerning the retracts defined using the Morse function. The reader is advised to compare these to Gromov's original work.

The novelty of our proof is the approach used to deal with elliptic elements. We adapt the Morse function to these elements in a way that takes into account their orders. This allows us to use the results of Section 4 to prove that the function is proper. Furthermore, this assures that sets of critical points are either short geodesics, or are contained in the quasi-thick parts of certain singular submanifolds. Using again the results of Section 4, we can bound the contribution of the latter sets to homology. The number of these sets is bounded using Theorems 3.2 and 3.5 (replacing the bound of Theorem 12.11 in [3]).

We will make use of the following lemma, proved in [3, Section 12.6].

Lemma 5.2 There are constants $\delta>0$ and $M_{1} \in \mathbb{N}$ such that for every $x, y \in X$, if $d(x, y)<2 \varepsilon_{n}$ and $\gamma \in \operatorname{Isom}(X)$ with $d_{\gamma}(x) \leq \delta$, then $d_{\gamma^{i}}(y) \leq \varepsilon_{n}$ for some $i \leq M_{1}$.

Proof of Theorem 5.1 We fix some constants, all of which depend only on $n$ :

$\varepsilon=\varepsilon_{n}, m=m_{n}$, the constants of the Margulis Lemma.

$M_{1}$ and $\delta$, the constants stipulated in Lemma 5.2. $M_{2}=m M_{1}$.

$J=M_{2}^{*}$ (following the notation of Proposition 2.5).

Our first step in the proof is to define a smooth function on $X / \Gamma$ through which we will study the topology of $X / \Gamma$.

We begin by fixing smooth functions

$$
g_{n}: \mathbb{R}^{\geq 0} \rightarrow \mathbb{R}^{\geq 0} \quad(n \geq 2) \quad \text { and } \quad g_{\infty}: \mathbb{R}^{>0} \rightarrow \mathbb{R}^{\geq 0}
$$

with the following properties:

- strictly decreasing on $(0, \varepsilon)$,

- identically zero on $[\varepsilon, \infty)$,

- $\quad g_{n}(0)=n$ and $g_{n}(\delta)=1$ for $n \geq 2$,

- $\lim _{x \rightarrow 0} g_{\infty}(x)=\infty$ and $g_{\infty}(\delta)=1$. 
We also require $g_{\infty}(\varepsilon / 2)$ and $g_{n}(\varepsilon / 2)$ to be smaller than some constant (computed later) that depends on $\Gamma$. We note that this will not affect any of the constants later defined (which do not depend on $\Gamma$ ).

For $1 \neq \gamma \in \Gamma$, let $o(\gamma) \in \mathbb{N} \cup\{\infty\}$ be the order of $\gamma$. We set $g_{\gamma}=g_{o(\gamma)}$.

We set

$$
\Delta=\left\{1 \neq \gamma \in \Gamma: \inf d_{\gamma}<\delta \text { and } \gamma \text { is } M_{2} \text {-stable if it is elliptic }\right\},
$$

and define

$$
F(x)=\sum_{\gamma \in \Delta} \sum_{i=0}^{M_{1}} g_{\gamma}\left(d_{\gamma^{i}}(x)\right) .
$$

This sum is locally finite because $\Gamma$ is discrete, and thus the set $\left\{\gamma \in \Gamma: d_{\gamma}(x)<2 \varepsilon\right\}$ is finite for every $x \in X$. Therefore, $F$ is a smooth function. Clearly, it is $\Gamma$-invariant, and thus descends to a smooth function (in the orbifold sense) $f$ on $X / \Gamma$.

It will be convenient to have a notation for elements in $\Gamma$ that contribute positive values to the sum defining $F$ at a given point. For $x \in X$ this is the set:

$$
\Delta_{x}=\left\{\gamma^{i}: \gamma \in \Delta, 1 \leq i \leq M_{1}, d_{\gamma^{i}}(x)<\varepsilon\right\}
$$

Note that if $\gamma^{\prime} \in \Delta_{x}$ and $\gamma^{\prime}=\gamma^{i}$ with $\gamma \in \Delta, i \leq M_{1}$, then $\operatorname{Min}(\gamma)=\operatorname{Min}\left(\gamma^{\prime}\right)$. Moreover, by the choice of $M_{2}$, every elliptic element of $\Delta_{x}$ is $m$-stable.

Claim $1 f$ is a proper map.

Proof We show that $f_{\leq r}$ is compact for every $r>0$. Fix $r>0$. Let $x \in X$ with $F(x) \leq r$. Suppose we have $\gamma \in \Gamma$ with $d_{\gamma}(x)<\delta$. If $\gamma$ is not elliptic then $\gamma \in \Delta_{x}$, and $F(x) \leq r$ implies $d_{\gamma}(x) \geq g_{\infty}^{-1}(r)$. Thus, if $\gamma$ has infinite order then $d_{\gamma}(x) \geq \min \left(\delta, g_{\infty}^{-1}(r)\right)=\mu_{1}$.

Set $\mu_{2}=\mu_{1} /(2 m J)$. We claim that $\Gamma_{\mu_{2}}(x)$ is finite. Indeed, if it is not then by Lemma 2.3, it would contain an element $\gamma$ of infinite order such that $d_{\gamma}(x)<2 m \mu_{2}<$ $\mu_{1}$, a contradiction.

Moreover, we may bound the order of $\Gamma_{\mu_{2}}(x)$ by a constant depending on $r$. Let $N$ be the normal abelian subgroup of $\Gamma_{\mu_{2}}(x)$. Let $\gamma$ be a generator of $N$ with $d_{\gamma}(x)<2 m \mu_{2}$. By the choice of $J$, there is some $j<J$ for which $\gamma^{j}$ is $M_{2}$-stable. Then $d_{\gamma^{j}}(x) \leq J d_{\gamma}(x)<J \cdot 2 m \mu_{2} \leq \delta$. Now, since $F(x) \leq r$, we have by the definitions of $F$ and of $g_{\gamma}$ that

$$
\operatorname{ord}\left(\gamma^{j}\right)=g_{\gamma^{j}}(\delta) \leq g_{\gamma^{j}}\left(d_{\gamma^{j}}(x)\right) \leq r
$$


and thus $\operatorname{ord}(\gamma) \leq J r$.

Therefore, the generators of $N$ have order bounded by $J r$, and since $N$ is abelian, the order of any element in $N$ is bounded by $(J r)$ !. Since $N$ is an abelian finite subgroup of $\mathrm{SO}(n)$, it is generated by at most $n$ elements (not necessarily those taken before), and hence $|N| \leq((J r) !)^{n}$ and $\left|\Gamma_{\mu_{2}}(x)\right| \leq m((J r) !)^{n}$.

Our discussion shows that $f_{\leq r}$ is contained in the $\left(m((J r) !)^{n}, \mu_{2}\right)$-quasi-thick part of $X / \Gamma$. By the assumption of finite essential volume of $X / \Gamma$, it follows by Corollary 4.3 that this quasi-thick part is compact.

Claim 2 A point $x \in X$ is a critical point of $F$ iff $x \in \bigcap_{\gamma \in \Delta_{x}} \operatorname{Min}(\gamma)$ (in particular, if $x$ is critical then $\Delta_{x}$ consists of semisimple elements). Moreover, if $x$ is not critical, and $A \subset \Delta_{x}$ is a set such that $x \in \operatorname{Min}(\gamma)$ for each $\gamma \in A$, then $\operatorname{grad} F$ is not perpendicular to $\bigcap_{\gamma \in A} \operatorname{Min}(\gamma)$.

Proof Assume $x \in \bigcap_{\gamma \in \Delta_{x}} \operatorname{Min}(\gamma)$. Let $\gamma \in \Delta$, and $1 \leq i \leq M_{1}$. If $\gamma^{i} \in \Delta_{x}$ then $x \in \operatorname{Min}\left(\gamma^{i}\right)$ and hence $\nabla d_{\gamma^{i}}(x)=0$. Otherwise, $d_{\gamma^{i}}(x) \geq \varepsilon$ and $g_{\gamma}^{\prime}\left(d_{\gamma^{i}}(x)\right)=0$. Hence, $x$ is critical because

$$
\nabla F(x)=\sum_{\gamma \in \Delta} \sum_{i=0}^{M_{1}} g_{\gamma}^{\prime}\left(d_{\gamma^{i}}(x)\right) \nabla d_{\gamma^{i}}(x)=0 .
$$

To prove the converse, suppose $x \notin \bigcap_{\gamma \in \Delta_{x}} \operatorname{Min}(\gamma)$. We shall find a geodesic ray $c:[0, \infty) \rightarrow X$ with $c(0)=x$ such that $\left.\frac{d}{d t} d_{\gamma}(c(t))\right|_{t=0} \leq 0$ for every $\gamma \in \Delta_{x}$ and such that $\left.\frac{d}{d t} d_{\gamma}(c(t))\right|_{t=0}<0$ for at least one $\gamma \in \Delta_{x}$. Once such a geodesic is found, we derive by equation (6) that

$$
\left.\frac{d}{d t} F(a(t))\right|_{t=0}>0
$$

(recall $g_{n}, g_{\infty}$ are strictly decreasing on $(0, \varepsilon)$ ), whence we deduce $\nabla F(x) \neq 0$. This will conclude the proof.

Let $\Gamma(x)=\left\langle\Delta_{x}\right\rangle$. Recall that elliptic elements of $\Delta_{x}$ are $m$-stable. Let $N(x)$ be the normal nilpotent subgroup of index $i \leq m$ in $\Gamma(x)$.

Suppose first that $N(x)$ does not contain parabolic elements. In this case, take $K=$ $\bigcap_{\gamma \in N(x)} \operatorname{Min}(\gamma)$. This is a convex $\Gamma(x)$-invariant set, and by Lemma 2.2, it is nonempty. By our assumption, there exists $\gamma \in \Delta_{x}$ such that $x \notin \operatorname{Min}(\gamma)$. Now, $\gamma$ is hyperbolic or elliptic and $m$-stable, and in either case, $\operatorname{Min}(\gamma)=\operatorname{Min}\left(\gamma^{i}\right)$. Since $\gamma^{i} \in N(x)$, we deduce that $x \notin K$. Let $c$ be the geodesic ray with $c(0)=x$ and $c(1)=\pi_{K}(x)$. Clearly, $d_{\gamma}(c(1))<d_{\gamma}(c(0))$ and since $d_{\gamma}$ is convex, it follows that 
$\left.\frac{d}{d t} d_{\gamma}(c(t))\right|_{t=0}<0$. On the other hand, any other $\gamma \in \Delta_{x}$ keeps $K$ invariant, and thus $d_{\gamma}(c(1)) \leq d_{\gamma}(c(0))$ and by convexity of $d_{\gamma},\left.\frac{d}{d t} d_{\gamma}(c(t))\right|_{t=0} \leq 0$.

If $N(x)$ does contain a parabolic, we claim that it contains a central parabolic. Indeed, since $N(x)$ is infinite, it has an infinite center, and thus has a central element of infinite order. But this element cannot be hyperbolic, otherwise we would have a parabolic element keeping a geodesic invariant (the axis of the central hyperbolic). Thus, all parabolic central elements in $N(x)$ fix a unique point $\xi \in \partial X$, the boundary of points at infinity. It follows that every element of $\Gamma(x)$ fixes $\xi$. Let $c$ be the geodesic ray with $c(0)=x, c(\infty)=\xi$. Each $\gamma \in \Delta_{x}$ keeps $\xi$ fixed, hence $d_{\gamma}$ is bounded on $c$, and by convexity:

$$
\left.\frac{d}{d t} d_{\gamma}(c(t))\right|_{t=0} \leq 0
$$

We claim that for at least one $\gamma \in \Delta_{x}$ this inequality is strict. Otherwise, $d_{\gamma}$ is constant on $c$ for all $\gamma \in \Delta_{x}$. This implies that $\gamma c \| c$, which is only possible if $\gamma$ fixes $c$. This contradicts the assumption that there exists $\gamma \in \Delta_{x}$ for which $x \notin \operatorname{Min}(\gamma)$.

To prove the last assertion in the claim, suppose $x$ is not critical, and $A \subset \Delta_{x}$ is a set such that $x \in \operatorname{Min}(\gamma)$ for each $\gamma \in A$. The geodesic we defined above has the property that $\left.\frac{d}{d t} d_{\gamma}(c(t))\right|_{t=0} \leq 0$ for every $\gamma \in \Delta_{x}$. Thus, $c$ is contained in $\operatorname{Min}(\gamma)$ for every $\gamma \in A$. Hence $\operatorname{grad} F$ is not perpendicular to $\bigcap_{\gamma \in A} \operatorname{Min}(\gamma)$.

Let $x$ be a critical point of $F$. We denote:

$$
Y_{x}=\bigcap_{\gamma \in \Delta_{x}} \operatorname{Min}(\gamma) \text { and } C_{x}=\left\{y \in Y_{x}: \Delta_{y}=\Delta_{x}\right\}
$$

By Claim 2, $x \in C_{x}$, and $C_{x}$ consists of critical points of $F$. Clearly, $F(x)$ is the minimum of $F$ on $Y_{x}$, and $C_{x}=F^{-1}(F(x)) \cap Y_{x}$. Note, also, that if $y \in C_{x}$ then $Y_{y}=Y_{x}$ and $C_{y}=C_{x}$. We call $\left(Y_{x}, C_{x}\right)$ a critical pair. It follows from the above that the critical pair is independent of a choice of critical point within the critical set. For a critical pair $(Y, C)$, we denote $\Delta_{C}=\Delta_{x}$ for some (hence any) $x \in C$.

Claim 3 Let $(Y, C)$ be a critical pair, and let $x \in X$.

(i) If $x$ is a critical point and $d(C, x)<2 \varepsilon$, then $Y_{x}=Y$ and $x \in C$.

(ii) If $d(x, C)<\varepsilon / 2$ and $d(\alpha x, C)<3 \varepsilon / 2$ for some $\alpha \in \Gamma$ then $\alpha$ keeps $Y$ invariant.

(iii) If $d(x, C)<\varepsilon / 2$ then $\Gamma_{\varepsilon}(x)$ keeps $Y$ invariant. 
Proof (i) Take $y \in C$ with $d(x, y)<2 \varepsilon$. Let $\gamma^{i} \in \Delta y$, with $\gamma \in \Delta$ and $1 \leq i \leq M_{1}$. Then $y \in \operatorname{Min}\left(\gamma^{i}\right)=\operatorname{Min}(\gamma)$ (stability is used if $\gamma$ is elliptic), hence $d_{\gamma}(y)<\delta$. By Lemma 5.2, $d_{\gamma^{j}}(x)<\varepsilon$ for some $j \leq M_{1}$, and thus $\gamma^{j} \in \Delta_{x}$. Since $x$ is critical, we have $x \in \operatorname{Min}\left(\gamma^{j}\right)=\operatorname{Min}\left(\gamma^{i}\right)$. Thus $d_{\gamma^{i}}(x)<\varepsilon$ and $\gamma^{i} \in \Delta_{x}$. We have proved $\Delta_{y} \subseteq \Delta_{x}$. The situation is symmetric to exchanging $x$ and $y$, and so $\Delta_{x}=\Delta_{y}$. Hence $Y_{x}=Y_{y}=Y$ and $x \in C$.

To prove (ii), take $y \in C$ with $d(x, y)<\varepsilon / 2$. Since $d(\alpha x, C)<3 \varepsilon / 2$, we have that $d(\alpha y, C)<2 \varepsilon$. Since $\alpha y$ is critical, we derive that $\alpha Y=Y_{\alpha y}=Y$ by (i).

(iii) follows from (ii). Indeed, if $\gamma \in \Gamma_{\varepsilon}(x)$ is a generator with $d_{\gamma}(x)<\varepsilon$ then $d(\gamma x, C)<3 \varepsilon / 2$.

Let us state an immediate corollary of this claim. Set:

$$
C^{\prime}=\{y \in Y: d(y, C)<\varepsilon / 2\}
$$

Note that $C^{\prime}$ is $\Gamma_{Y}$-invariant. By Claim 3 (ii), if $x \in C^{\prime}$ and $\gamma x \in C^{\prime}$ for some $\gamma \in \Gamma$ then $\gamma \in \Gamma_{Y}$. Hence:

Claim $4 C^{\prime} / \Gamma_{Y}$ injects into $X / \Gamma$.

Claim 5 For every $x \in Y, x$ is a critical point of $F$ if and only if it is a critical point of $\left.F\right|_{Y}$.

Proof Let $x \in Y$. It is trivial that if $x$ is a critical point of $F$ it is a critical point of $\left.F\right|_{Y}$. If $x$ is not a critical point of $F$, then by Claim 2, grad $F$ is not perpendicular to $Y=\bigcap_{\gamma \in \Delta_{C}} \operatorname{Min}(\gamma)$. Hence, $x$ is not a critical point of $\left.F\right|_{Y}$.

Claim 6 Let $x \in C^{\prime}$ and let $c:[0, \infty) \rightarrow X$ be a geodesic ray emanating from $x$ perpendicularly to $Y$. Then $F(c(t))$ is strictly decreasing for $t$ close to 0 .

Proof By Claim 3 (iii), elements of $\Delta_{x}$ keep $Y$ invariant, and thus for every $\gamma \in \Delta_{x}$, $\left.\frac{d}{d t} d_{\gamma}(c(t))\right|_{t=0} \geq 0$. It follows that $\left.\frac{d}{d t} F(c(t))\right|_{t=0} \leq 0$. Since $c$ is not contained in $Y$, there exists $\gamma \in \Delta_{C}$ for which $\left.\frac{d}{d t} d_{\gamma}(c(t))\right|_{t=0}>0$. Hence $\left.\frac{d}{d t} F(c(t))\right|_{t=0}<0$ for $t$ close to 0 .

At this stage, let us introduce some additional constants (all of which depend only on $n)$ :

$\delta_{2}=\frac{\delta}{2 m J+2}$ 
$\delta_{3}$ is the constant given by Theorem 4.2 for the $\left(\delta_{2}, m(J !)^{n}\right)$-quasi-thick part of an orbifold of dimension $\leq n$ (cf the remark following the theorem).

$\delta_{4}$ is the constant given by Theorem 3.2 for $\delta_{3}$.

$M_{3}=m(J !)^{n}$.

Claim 7 If $\Delta_{C}$ contains a hyperbolic element then $C=Y$ and $Y$ is a geodesic. Otherwise, $Y$ is an $m$-stable singular submanifold and there exists a $\Gamma_{Y}$-invariant neighborhood $C^{\prime \prime} \subset C^{\prime}$ of $C$ in $Y$ such that $C^{\prime \prime} / \Gamma_{Y}$ is contained in the $\left(\delta_{2}, M_{3}\right)$ quasi-thick part of $Y / \Gamma_{Y}$.

Proof Suppose first that $\Delta_{C}$ contains a hyperbolic element $\alpha$, with a geodesic axis $a$. Pick $x \in Y$ (not necessarily in $C$ ). Then $\alpha \in \Delta_{x}$, and thus $\bigcap_{\gamma \in \Delta_{x}} \subseteq a$. Let $\Gamma(x)=\left\langle\Delta_{x}\right\rangle$ and let $N$ be the normal nilpotent subgroup of $\Gamma(x)$ of index $i<m$. Let $H$ be the set of semisimple elements in $N$. Then $H$ is a normal subgroup of $N$ and $Z=\bigcap_{\gamma \in H} \operatorname{Min}(\gamma)$ is non-empty, convex and is kept invariant by $H$. Since $\alpha^{i} \in H$, we deduce that $Z \subseteq a$, and since $Z$ is $\alpha^{i}$-invariant, this is only possible if $Z=a$. On the other hand, for every $\gamma \in \Delta_{x}, \gamma$ is semisimple and $\gamma^{i} \in N$, thus $\gamma^{i} \in H$. Hence $a \supseteq \bigcap_{\gamma \in \Delta_{x}} \operatorname{Min}(\gamma)=\bigcap_{\gamma \in \Delta_{x}} \operatorname{Min}\left(\gamma^{i}\right) \supseteq Z$. We deduce that $\bigcap_{\gamma \in \Delta_{x}} \operatorname{Min}(\gamma)=a$. Since this is true for every $x \in Y$, it follows that $C=Y=a$.

Suppose now that $\Delta_{C}$ consists only of elliptic elements. Since elements of $\Delta_{C}$ are $m$-stable, $Y$ is an $m$-stable singular submanifold. We take:

$$
C^{\prime \prime}=\left\{y \in Y: d(y, C)<\delta_{2}\right\}
$$

Let $y \in C^{\prime \prime}$ and pick $x \in C$ such that $d(x, y)<\delta_{2}$.

We first prove that $\Gamma_{\delta_{2}}(y)$ is finite. Suppose not. Then by Lemma 2.3, there is an element $\gamma \in \Gamma_{\delta_{2}}(y)$ of infinite order with $d_{\gamma}(y)<2 m \delta_{2}$. But then $d_{\gamma}(x)<$ $(2 m+2) \delta_{2}<\delta$ and hence $\gamma \in \Delta_{x}$, contradicting our assumption on $\Delta_{C}=\Delta_{x}$.

Since $\Gamma_{\delta_{2}}(y)$ is finite, it has a normal abelian subgroup $N$ of index $i<m$. Let $\gamma$ be a generator of $N$ with $d_{\gamma}(y)<2 m \delta_{2}$. There exists some $j<J$ such that $\gamma^{j}$ is $M_{2}-$ stable, by Proposition 2.5. But $d_{\gamma^{j}}(y)<2 m J \delta_{2}$, and thus $d_{\gamma^{j}}(x)<(2 m J+2) \delta_{2}=\delta$. Thus $\gamma^{j}=1$ or else $\gamma^{j} \in \Delta_{x}$. In either case, $\gamma^{j}$ fixes $Y$ pointwise. Hence, for every such generator of $N$ we have $\gamma^{J !} \in N \cap \Gamma_{Y}^{1}$. Since $N$ is abelian, we have that $\gamma^{J !} \in N \cap \Gamma_{Y}^{1}$ for all $\gamma \in N$. Since $N$ is finite and abelian, it is generated by at most $n$ elements, and thus $\left[N: N \cap \Gamma_{1}^{Y}\right]<(J !)^{n}$. Therefore $\Gamma_{\delta_{2}}(y)$ has at most $m(J !)^{n}=M_{3}$ elements. 
We turn now to the critical points of $f$. Every critical point of $f$ has a preimage in $X$ which is a critical point of $F$. Hence, we may choose a maximal collection of nonconjugate critical pairs $\left\{\left(Y_{i}, C_{i}\right)\right\}$ such that each critical point of $f$ is in exactly one projection $\pi\left(C_{i}\right)$. A consequence of Claim 7 is that there are only finitely many nonconjugate critical pairs, hence finitely many critical values of $f$. Indeed, Theorem 3.5 bounds the number of pairs $(Y, C)$ for which $\Delta_{C}$ contains a hyperbolic element. On the other hand, if $(Y, C)$ is a critical pair with $\Delta_{C}$ consisting of elliptic elements, then by Theorem 4.2, $\delta_{3}$-ess- $\operatorname{vol}\left(Y / \Gamma_{Y}\right) \geq 1$. Thus by Theorem 3.2, the number of such pairs is bounded by $\delta_{4}$-ess-vol $(X)$, and is finite, in particular. By Corollary 4.3, each of the sets $\pi\left(C_{i}\right)$-hence their union-is compact.

We wish to bound the values of $F$ in a neighborhood of $C$. Let $y \in Y$ with $d(y, C) \leq$ $\varepsilon / 4$. Pick $x \in C$ such that $d(x, y)<\varepsilon / 4$. Recall that $\Delta_{x} \subseteq \Delta_{y}$, and that the restriction of $F$ to $Y$ attains its minimum in $x$. Consider $\gamma \in \Delta_{y}$. If $\gamma \in \Delta_{x}$ then $d_{\gamma}(y)=d_{\gamma}(x)$. Otherwise, $d_{\gamma}(x) \geq \varepsilon$, and hence $d_{\gamma}(y) \geq \varepsilon / 2$. Therefore,

$$
F(y)-F(x) \leq K \cdot\left|\Delta_{y} \backslash \Delta_{x}\right|
$$

where $K=\max \left\{g_{i}(\varepsilon / 2)\right\}$ (recall the definition of the functions $g_{i}$ ). The set of points of distance $\leq \varepsilon / 4$ from union of the sets $\pi\left(C_{i}\right)$ is compact. Let $B$ be a compact preimage of this union in $X$. By compactness, there is a uniform upper bound (depending on $\Gamma$ ) on the size of $\Delta_{y}$ for every $y \in B$. Hence, we may require $g_{i}(\varepsilon / 2)$ to be sufficiently small so that

$$
F(y)-F(x) \leq \frac{1}{2}
$$

for every $x \in C$ and $y \in X$ with $d(y, C)<\varepsilon / 4$.

Let $0=c_{1}<c_{2}<\cdots<c_{k}$ be the distinct critical values of $f$. Let $\mu$ be small enough such that for every critical value $c, c$ is the only critical value in the interval $[c-\mu, c+\mu]$, and such that these intervals are disjoint for distinct critical values. Since $f$ is a proper map, a standard argument of Morse theory [14] shows that the flow along gradient lines defines a deformation retract of $X / \Gamma$ onto $f_{\leq c_{k}+\mu}$ and of $f_{\leq c_{i+1}-\mu}$ onto $f_{\leq c_{i}+\mu}$. Thus, since the rank of homology is subadditive for triples of spaces, we have:

$$
\operatorname{rk} H_{j}(X / \Gamma) \leq \sum_{i=1}^{k} \operatorname{rk} H_{j}\left(f_{\leq c_{i}+\mu}, f_{\leq c_{i}-\mu}\right)
$$

We will bound the rank of relative homology groups on the right-hand side of this inequality. Let $c$ be a critical value. Regarding $c$ as a critical value of $F$, it may be the case that there are several non-conjugate critical pairs corresponding to this value. 
However, since distinct critical sets are disjoint (by Claim 3), and since our analysis is local, we will assume $(Y, C)$ is a unique (up to conjugacy) critical pair with this critical value.

Denote $V=f_{\leq c+\mu} \cap \pi(Y)$. We assume $\mu$ is sufficiently small such that $V$ is contained in $\pi\left(C^{\prime \prime}\right)$. To bound rk $H_{j}\left(f_{\leq c+\mu}, f_{\leq c-\mu}\right)$, we consider the triple:

$$
f_{\leq c-\mu} \subseteq\left(f_{\leq c+\mu} \backslash V\right) \subseteq f_{\leq c+\mu}
$$

By Claim 6, the flow along $-(\operatorname{grad} f)$ near $\pi\left(C^{\prime \prime}\right)$ does not approach $\pi(Y)$, thus a flow along $-(\operatorname{grad} f)$ starting from a point in $f_{\leq c+\mu} \backslash V$ does not reach $\pi\left(C^{\prime \prime}\right)$. Since there are no critical points in this domain, this flow defines a deformation retract of $f_{\leq c+\mu} \backslash V$ to $f_{\leq c-\mu}$. Therefore:

$$
H_{j}\left(f_{\leq c+\mu} \backslash V, f_{\leq c-\mu}\right)=0
$$

By the long exact sequence associated to the triple (9), $H_{j}\left(f_{\leq c+\mu}, f_{\leq c-\mu}\right)$ is thus isomorphic to $H_{j}\left(f_{\leq c+\mu}, f_{\leq c+\mu} \backslash V\right)$. Now, by excision,

$$
H_{j}\left(f_{\leq c+\mu}, f_{\leq c+\mu} \backslash V\right) \simeq H_{j}(U, U \backslash V)
$$

where $U$ is some neighborhood of $V$ in $f_{\leq c+\mu}$. By the tubular neighborhood lemma, $N(V)$ - the (orbifold) normal bundle of $V$ in $X / \Gamma$-is diffeomorphic to a neighborhood of $V$ in $f_{\leq c+\mu}$. Thus we conclude that

$$
H_{j}\left(f_{c+\mu}, f_{c-\mu}\right) \simeq H_{j}\left(N(V), N(V) \backslash N_{0}(V)\right)
$$

where $N_{0}$ is the zero section of $N$.

The remainder of our analysis will be inside $Y$. Let us denote the restriction of $F$ to $Y$ by $\widetilde{F}$, and the restriction of $f$ to $Y / \Gamma$ by $\tilde{f}$. We are only interested in the behavior of these functions in $C^{\prime \prime}$ and $C^{\prime \prime} / \Gamma_{Y}$, correspondingly. Recall that we have chosen $\mu$ such that $V \subseteq \pi\left(C^{\prime \prime}\right)$. Also, recall that by Claim $4, C^{\prime \prime} / \Gamma_{Y}$ injects into $X / \Gamma$. We can therefore consider $V$ as a subset of $Y / \Gamma_{Y}$. By Claim 7 there are two possibilities: (1) $V=Y / \Gamma_{Y}$ is 1-dimensional or (2) $V$ is contained in the $\left(\delta_{2}, M_{3}\right)$-quasi-thick part of $Y / \Gamma_{Y}$.

If $V$ is 1-dimensional then it is either contractible, or is a circle. Either way:

$$
\operatorname{rk} H_{j}(N(V)) \leq 1 \quad \text { and } \quad \operatorname{rk} H_{j}\left(N(V) \backslash N_{0}(V)\right) \leq 2
$$

Thus:

$$
\text { rk } H_{j}\left(N(V), N(V) \backslash N_{0}(V)\right) \leq 3
$$

Otherwise, by Theorem 4.2, we can cover $V$ with a good cover of balls of radius $\leq \delta_{2}$, where the number of balls does not exceed $\delta_{3}$-ess-vol $\left(Y / \Gamma_{Y}\right)$. Let $U$ be the union of 
these balls. Since $N(U) \backslash N_{0}(U)$ is homotopy equivalent to a sphere bundle over $U$, Proposition 4.11 states that

$$
\text { rk } H_{j}(N(U)) \leq h(r) \cdot \delta_{3}-\operatorname{ess}-\operatorname{vol}\left(Y / \Gamma_{Y}\right)
$$

and

$$
\text { rk } H_{j}\left(N(U) \backslash N_{0}(U)\right) \leq h(r) \cdot \delta_{3}-\text { ess-vol }\left(Y / \Gamma_{Y}\right)
$$

where $r$ is the bound on the number of balls with non-empty intersection in the cover of $V$.

Since each ball in the cover has radius $\leq \delta_{2}$, it follows that $U \subset C^{\prime}$. Thus, by (7)

$$
U \subseteq \tilde{f}_{\leq c+\frac{1}{2}}
$$

We claim that $\tilde{f}$, and equivalently $\widetilde{F}$, has no critical values between $c+\mu$ and $c+\frac{1}{2}$. Suppose $y \in Y$ is a critical point of $\tilde{F}$, with $f(y) \geq c+\mu$. Then there exists some $\gamma^{\prime} \in \Delta_{x} \backslash \Delta_{C}$, and $\gamma^{\prime}=\gamma^{i}$ for some $\gamma \in \Delta$ and $1 \leq i \leq M_{1}$. By Claim 5, $y$ is also a critical point of $F$, hence by Claim 2, $x \in \operatorname{Min}\left(\gamma^{\prime}\right)$, and by stability, $x \in \operatorname{Min}(\gamma)$. It follows that $d_{\gamma}(x)<\delta$, and thus $g_{\gamma}\left(d_{\gamma}(x)\right)>1$. Therefore, $F(x) \geq c+1$ and the claim follows.

We conclude that:

$$
V=\tilde{f}_{\leq c+\mu} \subseteq U \subseteq \tilde{f}_{\leq c+\frac{1}{2}}
$$

Since there are no critical values between $c+\frac{1}{2}$ and $c+\mu, \tilde{f}_{\leq c+\mu}$ is a deformation retract of $\tilde{f}_{\leq c+\frac{1}{2}}$, and induces an isomorphism on homology. Thus the inclusion induces maps

$$
H_{j}(V) \rightarrow H_{j}(U) \rightarrow H_{j}\left(\tilde{f}_{\leq c+\frac{1}{2}}\right)
$$

whose composition is an isomorphism. Hence, $\operatorname{rk}\left(H_{j}(V)\right) \leq \mathrm{rk}\left(H_{j}(U)\right)$, and similarly, $\operatorname{rk}\left(H_{j}\left(N(V) \backslash N_{0}(V)\right)\right) \leq \operatorname{rk}\left(H_{j}\left(N(U) \backslash N_{0}(U)\right)\right)$. Thus:

$$
\text { rk } H_{j}\left(N(V), N(V) \backslash N_{0}(V)\right) \leq 2 h(r) \cdot \delta_{3}-\operatorname{ess}-\operatorname{vol}\left(Y / \Gamma_{Y}\right)
$$

Let $\delta_{5}$ be the constant stipulated in Theorem 3.5, used to bound the number of "short geodesics". Then pairs $(Y, C)$ such that $\Delta_{C}$ contains a hyperbolic element contribute at most $\delta_{5}$-ess-vol $(X / \Gamma)$ terms to the sum in (8). Each such term is $\leq 3$. The remaining terms correspond to non-conjugate $m$-stable singular manifolds. Each such term contributes $2 h(r) \cdot \delta_{3}$-ess-vol $\left(Y / \Gamma_{Y}\right)$. Hence, by Theorem 3.2, all these terms contribute

$$
\leq 2 h(r) \sum_{Y \in \Sigma} \delta_{3}-\mathrm{ess}-\operatorname{vol}\left(Y / \Gamma_{Y}\right) \leq 2 h(r) \cdot \delta_{4}-\operatorname{ess}-\operatorname{vol}(X / \Gamma)
$$


where $\Sigma$ is a set of non-conjugate $m$-stable singular submanifolds.

Thus, (8) yields:

$$
\begin{aligned}
\operatorname{rk} H_{j}(X / \Gamma) & \leq 3 \cdot \delta_{5}-\mathrm{ess}-\operatorname{vol}(X / \Gamma)+2 h(r) \cdot \delta_{4}-\mathrm{ess}-\operatorname{vol}(X / \Gamma) \\
& \leq(3+2 h(r))\left(\min \left(\delta_{5}, \delta_{5}\right)\right)-\mathrm{ess}-\operatorname{vol}(X / \Gamma) \\
& \leq \frac{3+2 h(r)}{\min \left(\delta_{5}, \delta_{5}\right)}-\mathrm{ess}-\operatorname{vol}(X / \Gamma)
\end{aligned}
$$

We take:

$$
v=\frac{3+2 h(r)}{(n+1) \min \left(\delta_{4}, \delta_{5}\right)}
$$

One easily verifies that all constants depend only on $n$. We have:

$$
\sum_{i=0}^{n} \operatorname{rk} H_{i}(X / \Gamma) \leq(n+1) \cdot((n+1) v)-\operatorname{ess}-\operatorname{vol}(X / \Gamma) \leq v-\operatorname{ess}-\operatorname{vol}(X / \Gamma)
$$

\section{References}

[1] M Abert, N Bergeron, I Biringer, T Gelander, N Nikolov, J Raimbault, I Samet, On the growth of $L^{2}$-invariants for sequences of lattices in Lie groups arXiv: 1210. 2961

[2] M Abert, N Bergeron, I Biringer, T Gelander, N Nikolov, J Raimbault, I Samet, On the growth of Betti numbers of locally symmetric spaces, C. R. Math. Acad. Sci. Paris 349 (2011) 831-835 MR2835886

[3] W Ballmann, M Gromov, V Schroeder, Manifolds of nonpositive curvature, Progress in Mathematics 61, Birkhäuser, Boston, MA (1985) MR823981

[4] G Baumslag, Lecture notes on nilpotent groups, Regional Conference Series in Mathematics 2, American Mathematical Society (1971) MR0283082

[5] M R Bridson, A Haefliger, Metric spaces of non-positive curvature, Grundl. Math. Wissen. 319, Springer, Berlin (1999) MR1744486

[6] KS Brown, Cohomology of groups, Graduate Texts in Mathematics 87, Springer, New York (1982) MR672956

[7] E E Floyd, Periodic maps via Smith theory, from: "Seminar on transformation groups", (A Borel, editor), Annals of Mathematics Studies 46, Princeton University Press (1960) 35-47

[8] T Gelander, Homotopy type and volume of locally symmetric manifolds, Duke Math. J. 124 (2004) 459-515 MR2084613 
[9] T Gelander, Volume versus rank of lattices, J. Reine Angew. Math. 661 (2011) 237-248 MR2863908

[10] M Gromov, Volume and bounded cohomology, Inst. Hautes Études Sci. Publ. Math. (1982) 5-99 MR686042

[11] C Jordan, Mémoire sur les équations différentielles linéaires à intégrale algébrique, J. Reine Angew. Math. 84 (1878) 89-215

[12] W Lück, Approximating L $\langle 2\rangle$-invariants by their finite-dimensional analogues, Geom. Funct. Anal. 4 (1994) 455-481 MR1280122

[13] W Magnus, A Karrass, D Solitar, Combinatorial group theory: presentations of groups in terms of generators and relations, 2nd edition, Dover Publications, New York (1976) MR0422434

[14] J Milnor, Morse theory, Annals of Mathematics Studies 51, Princeton Univ. Press (1963) MR0163331

[15] M Olbrich, L〈2〉-invariants of locally symmetric spaces, Doc. Math. 7 (2002) 219-237 MR1938121

[16] MS Raghunathan, Discrete subgroups of Lie groups, Ergebnisse der Mathematik und ihrer Grenzgebiete 68, Springer, New York (1972) MR0507234

[17] I Satake, On a generalization of the notion of manifold, Proc. Nat. Acad. Sci. U.S.A. 42 (1956) 359-363 MR0079769

[18] V Schroeder, Finite volume and fundamental group on manifolds of negative curvature, J. Differential Geom. 20 (1984) 175-183 MR772130

[19] N Steenrod, The topology of fibre bundles, Princeton Mathematical Series 14, Princeton Univ. Press (1951) MR0039258

[20] W P Thurston, The geometry and topology of three-manifolds (1980) Lecture notes from Princeton University Available at http://library.msri.org/books/gt3m

Department of Mathematics, Statistics, and Computer Science, University of Illinois at Chicago Chicago, IL 60607, USA

samet@math . uic . edu

Proposed: Walter Neumann

Received: 27 September 2011

Seconded: Dmitri Burago, John Lott

Revised: 8 Nov 12 